\title{
Magnetic field oscillations near the planetary period in Saturn's equatorial magnetosphere: Variation of amplitude and phase with radial distance and local time
}

\author{
D. J. Andrews, ${ }^{1}$ S. W. H. Cowley, ${ }^{1}$ M. K. Dougherty, ${ }^{2}$ and G. Provan ${ }^{1}$ \\ Received 5 August 2009; revised 5 October 2009; accepted 18 November 2009; published 22 April 2010.
}

[1] We present an analysis of the $\sim 11 \mathrm{~h}$ oscillations in Saturn's near-equatorial magnetic field, using Cassini data acquired during 2004-2007. We assume the oscillation period is given by the magnetic phase model derived by Provan et al. (2009) over the same interval, and use this to combine the data to determine the variation of the oscillation amplitude and phase of all three spherical polar field components with radial distance $\left(\sim 3-30 R_{S}\right)$ and local time $\left(\mathrm{R}_{\mathrm{S}}\right.$ is Saturn's radius, $\left.60,268 \mathrm{~km}\right)$. The oscillatory field behavior can be divided into two regions at a radial distance of $\sim 15 \mathrm{R}_{\mathrm{S}}$. In the inner region the radial and azimuthal components form a rotating field that to a first approximation is quasi-uniform, but shows major suppression and deflection effects around the near-planet region.

Associated rotating field-aligned currents in the Enceladus torus are estimated to carry $\sim \pm 1$ MA. In the outer region these field components form a rotating partial twin-vortex centered in the nightside, with associated North-South directed currents carrying $\sim \pm 6$ MA. Individual current regions of a given sign emerge first at dusk, propagate via midnight, and dissipate near dawn, avoiding the dayside sector of weaker more uniform oscillatory fields. Oscillations in the colatitudinal field are also present throughout, that are generally in phase with the radial component. The oscillation phases of all components are found to increase with radial distance at all local times, indicating outward radial propagation with phase speeds of $\sim 200 \mathrm{~km} \mathrm{~s}^{-1}$ on the nightside and $\sim 500 \mathrm{~km} \mathrm{~s}^{-1}$ on the dayside.

Citation: Andrews, D. J., S. W. H. Cowley, M. K. Dougherty, and G. Provan (2010), Magnetic field oscillations near the planetary period in Saturn's equatorial magnetosphere: Variation of amplitude and phase with radial distance and local time, J. Geophys. Res., 115, A04212, doi:10.1029/2009JA014729.

\section{Introduction}

[2] Saturn's internally-generated magnetic field has been shown to be highly axisymmetric, such that to date only an upper limit of $0.5^{\circ}$ has been set on the tilt between the planet's spin and magnetic axes [Smith et al., 1980; Connerney et al., 1982; Davis and Smith, 1990; Giampieri et al., 2006]. Despite this, many magnetospheric phenomena have been found to exhibit oscillations close to the $\sim 11 \mathrm{~h}$ planetary rotation period, notably the Saturn kilometric radiation (SKR) emission discovered in Voyager radio wave data, that is believed to be generated by precipitating auroral electrons [Warwick et al., 1981; Lecacheux and Genova, 1983; Galopeau et al., 1995; Zarka, 1998]. The modulation in this emission has been found to be independent of the position of the observer, thus being termed "strobe like." Initially, the SKR modulation was used to define Saturn's internal rotation period, based on the assumption that it is due to the presence of some anomaly (e.g., magnetic) that

\footnotetext{
${ }^{1}$ University of Leicester, Leicester, UK.

${ }^{2}$ Blackett Laboratory, Imperial College, London, UK.
}

Copyright 2010 by the American Geophysical Union. 0148-0227/10/2009JA014729 rotates rigidly with the planet [Desch and Kaiser, 1981]. However, subsequent analyses using Ulysses and Cassini data have shown that the SKR modulation period is variable, changing by of order $\sim 1 \%$ over yearly intervals [Galopeau and Lecacheux, 2000; Gurnett et al., 2005]. Kurth et al. [2007, 2008] have determined the phase of the SKR modulation using Cassini radio data from January 2004 to August 2007, and found the modulation period increased monotonically over this interval from $\sim 10.76$ to $\sim 10.84 \mathrm{~h}$. Such changes are much too large to reflect variations in the rotation rate of the deep interior of the planet, and must instead relate to the periodicity in some outer gaseous region. Here, therefore, we refer to these modulations as being "magnetospheric period oscillations" rather than "planetary period oscillations."

[3] Modulations near the magnetospheric period were also detected in the in situ charged particle and magnetic field data obtained during the Pioneer-11 and Voyager-1 and -2 flybys [Carbary and Krimigis, 1982; Espinosa and Dougherty, 2000; Espinosa et al., 2003a, 2003b], and have now been investigated in more detail using Cassini data [e.g., Krupp et al., 2005; Clarke et al., 2006; Gurnett et al., 2007; Carbary et al., 2007a, 2007b, 2008a, 2008b; Burch et al., 2009]. It is important to note that the oscillations in these 
phenomena are not strobe like, but rather rotate around the planet at the magnetospheric period as an " $m=1$ " perturbation, where $m$ is the azimuthal wave number. The phase of these oscillations thus varies with the azimuth (or equivalently local time, LT) of the observer, and variable Doppler shifts are observed due to the azimuthal motion of the spacecraft through the wavefield [Cowley et al., 2006; Giampieri et al., 2006; Carbary et al., 2007c; Southwood and Kivelson, 2007]. Evidence has also been presented for outward radial propagation of these modulations, such that the overall phase fronts of these disturbances form spiral structures in and near the equatorial plane [Cowley et al., 2006; Gurnett et al., 2007; Carbary et al., 2007b].

[4] In this paper we will primarily be concerned with the oscillations in the magnetic field in the near-equatorial magnetosphere. Previous studies have shown that within the quasi-dipolar core region of the magnetosphere, out to equatorial radial distances of $\sim 12 \mathrm{R}_{\mathrm{S}}$ (where the planetary radius $1 \mathrm{R}_{\mathrm{S}}$ is equal to $60,268 \mathrm{~km}$ ), the perturbations in the spherical polar radial $(r)$ and azimuthal $(\varphi)$ field components take the form of a rotating quasi-uniform field of $\sim 1-2 \mathrm{nT}$ amplitude, which does not vary strongly with colatitude about the equator [Espinosa et al., 2003a, 2003b; Southwood and Kivelson, 2007; Andrews et al., 2008]. Expressed in terms of the relative phase of these field components, the azimuthal component oscillations are consistently in lagging quadrature with the radial component oscillations. Neither this polarization, nor the weak amplitude dependence on radial distance is consistent with a rotating asymmetric source in the planet's internally generated field, the amplitude of which would fall off with radius as $r^{-3}$ or faster. Instead, the field perturbations must thus be associated with the same external current system. With regard to the connection with the SKR modulation, it has been found that the emission power peaks when the rotating field points toward $\sim 2 \pm \mathrm{hLT}$, i.e., tailward and dawnward. In addition to the oscillations in the radial and azimuthal field components, it has been found that oscillations of comparable amplitude are also present in the colatitudinal $(\theta)$ field component in the core region [Andrews et al., 2008; Khurana et al., 2009]. These field oscillations in the North-South component have been found to be in phase with the radial component oscillations in this region.

[5] An empirical model of the phase of the magnetic field oscillations within the core region has been determined by Andrews et al. [2008] using data from 23 near-equatorial periapsis passes during the early part of the Cassini orbital mission, spanning October 2004 to July 2006. A crosscorrelation technique was used that accounted for Doppler shifting of the oscillation due to the azimuthal motion of the spacecraft, and employed the SKR phase model of Kurth et al. [2007] as an exact "guide phase" with which to track the variation of the magnetic phase from pass to pass. Subsequently, Provan et al. [2009a] used the same technique together with the Kurth et al. [2008] SKR phase model to analyze a longer interval of magnetic field data, thereby deriving a magnetic phase model between July 2004 and December 2007. These studies showed that the core region field oscillations and the SKR modulation exhibit a common, consistent period over the $\sim 3$ year interval of joint data examined, with a phase difference that remains approximately fixed within the (often considerable) scatter in the SKR data. This magnetic field phase model was used by Provan et al. [2009a] to study related field oscillations observed by Cassini at high latitudes on open field lines, while Provan et al. [2009b] also employed the model to study the phase of the magnetospheric period oscillations of Saturn's auroral oval discovered by Nichols et al. [2008]. The Provan et al. [2009a] magnetic phase model also provides the basis for the analysis presented here.

[6] Theoretical consideration of these oscillatory phenomena have centered on discussion of "corotating convection" systems driven by plasma mass-loading in the inner magnetosphere originating from the icy moon Enceladus [Gurnett et al., 2007; Goldreich and Farmer, 2007]. The system of currents perpendicular and parallel to the magnetic field that couples the momentum in such flows to and from the ionosphere is generally antisymmetric about the equator, and produces antisymmetric field perturbations unlike the symmetric quasi-uniform field observed within the core region. Instead, the observed field suggests the presence of net field-aligned currents flowing from hemisphere to hemisphere outside the core, that produce a quasi-uniform field inside the current system as observed, and a quasidipolar field transverse to the planetary dipole on the outside [Southwood and Kivelson, 2007]. However, as discussed by Provan et al. [2009a], such currents could in effect be generated by a corotating convection system due to seasonal asymmetries in the northern and southern field-aligned currents, arising, for example, from seasonal asymmetries in the northern and southern ionospheric Pedersen conductivities. We note that the early part of the Cassini mission corresponded to southern summer and northern winter conditions at Saturn. Even so, it is not obvious that the relative phasing between the inner plasma density variations and the magnetic field oscillations found by Gurnett et al. [2007] can be explained by this physical picture [Andrews et al., 2008; Provan et al., 2009a].

[7] An alternative theoretical picture has been presented by Khurana et al. [2009], in which the oscillatory phenomena are instead proposed to be due to the seasonally-dependent oblique flow of the solar wind around the magnetosphere, acting upon a rotating asymmetric ring current plasma population. However, while these authors discussed the oscillations in the colatitudinal field that would be produced by the rotating asymmetric ring current plasma, and related these to oscillations of the tail plasma sheet, they offered no explanation of the simultaneous oscillations in the radial and azimuthal field components discussed above. It thus remains unclear whether this scenario provides an overall explanation for the magnetic field phenomena observed.

[8] In this paper we contribute to these studies by building on the analyses of Andrews et al. [2008] and Provan et al. [2009a] to examine how the oscillations in Saturn's equatorial magnetic field depend both on radial distance and LT from the inner region out to $\sim 30 \mathrm{R}_{\mathrm{S}}$, thus extending the investigation well beyond the equatorial core region examined previously. In addition, for the first time we determine not only the phase of the oscillations, but also their amplitude throughout this region. From these data we can also derive the pattern of oscillatory currents flowing NorthSouth through the equatorial plane, and compare these with the theoretical pictures proposed, e.g., by Southwood and Kivelson [2007] and Provan et al. [2009a]. In section 2 


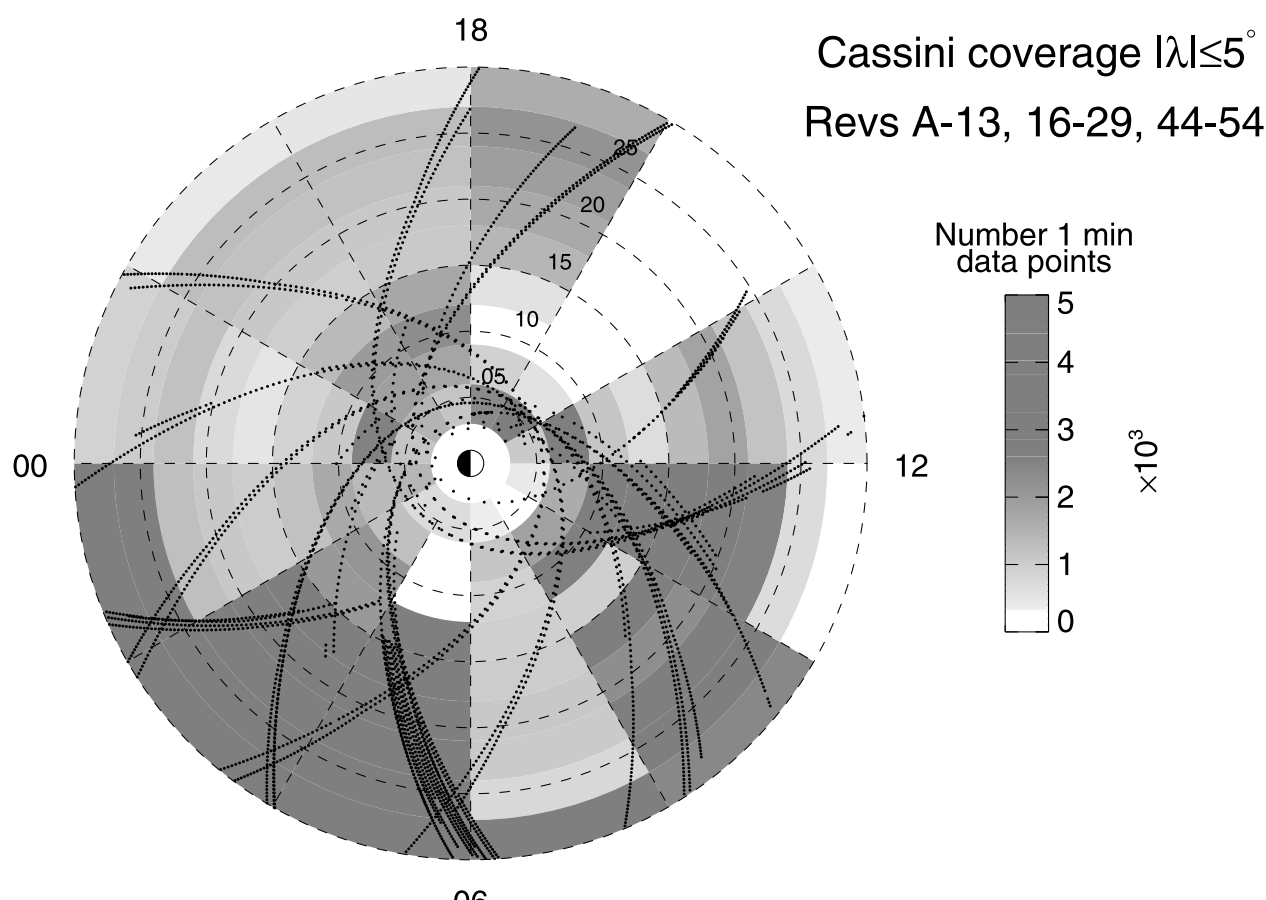

06

Figure 1. Plot of the Cassini coverage of Saturn's equatorial region during the interval of this study. The view is from the North looking down on to the equatorial plane, with the Sun to the right. LT is indicated around the perimeter of the plot, with dashed lines at $2 \mathrm{~h}$ intervals. The outer radius of the plot corresponds to $30 R_{S}$, with dashed circles being shown every $5 R_{S}$. Each black point indicates the spacecraft position projected onto the equatorial plane at $1 \mathrm{~h}$ intervals when the spacecraft is located at latitudes within $\pm 5^{\circ}$ of the equator, and magnetic data are available. The grey-scale coding indicates the number of 1 min averaged magnetic field measurements that are available within nonoverlapping spatial bins of $3 \mathrm{R}_{\mathrm{S}}$ in radial distance and $2 \mathrm{~h}$ in $\mathrm{LT}$, as indicated by the scale on the right. Each bin typically contains $\sim 1000-5000$ points, giving good coverage outside of $\sim 3 \mathrm{R}_{\mathrm{S}}$ at all LTs, except at 1200-1400 LT beyond $\sim 7 \mathrm{R}_{\mathrm{S}}$, and $0400-0600 \mathrm{LT}$ in the $\sim 6-12 \mathrm{R}_{\mathrm{S}}$ radial range.

we begin by describing the Cassini data set employed, together with the method of analysis.

\section{Data Selection and Analysis}

\subsection{Cassini Equatorial Data Coverage}

[9] Our initial data set consists of 1 min averaged magnetic field data in spherical polar coordinates referenced to the planet's spin axis, that were obtained by the Cassini fluxgate magnetometer [Dougherty et al., 2004]. These data were obtained during the interval from July 2004 to December 2007, corresponding to the 56 orbits designated "Saturn orbit insertion" (SOI) through to the end of Rev 54. During this interval the spacecraft was predominantly orbiting near Saturn's equatorial plane such that the data obtained are well suited to a comprehensive analysis of the magnetospheric period field oscillations in the nearequatorial region, defined here to be the region within $\pm 5^{\circ}$ latitude of the planet's equator. However, on Revs SOI and 30-43 the spacecraft trajectory was significantly tilted with respect to the equatorial plane, thus providing insufficient contiguous data within this region for the study of $\sim 11 \mathrm{~h}$ oscillations. The data for these orbits have thus been removed from our data set. Revs 14 and 15 are further excluded due to the presence of substantial gaps in the magnetometer data during the spacecraft's passage through the region considered. The volume of data from the remaining 39 orbits is largely insensitive to the latitude cutoff employed, since the trajectories of these orbits generally lie close to the equatorial plane within a few degrees. Extending the latitude cutoff to $\pm 10^{\circ}$, for example, does not greatly expand the data set available or its coverage in radial distance and local time.

[10] We note that this set of Cassini orbits is effectively the same as that employed by Provan et al. [2009a] to study the phase of the field oscillations within the quasi-dipolar core region, defined in that study as dipole $L \leq 12$. However, here we set the outer edge at a radial distance of $30 R_{S}$ so that we cover a significantly larger region, extending from the vicinity of the magnetopause on the dayside into the inner tail on the nightside. The region studied here, therefore, extends beyond the quasi-dipolar core into the region where the near-equatorial field forms a "magnetodisc" structure [Arridge et al., 2008], merging into the tail plasma sheet on the nightside. Since radial distances of $30 R_{S}$ can also sometimes extend beyond the magnetopause on the dayside, we have examined these data by hand to remove all magnetosheath and solar wind data, as informed by the magnetopause model of Arridge et al. [2006]. The innermost radial range sampled on these orbits is typically $\sim 3 R_{S}$.

[11] The data employed here were thus obtained on Revs A-13 (August 2004 to August 2005), 16-29 (October 2005 


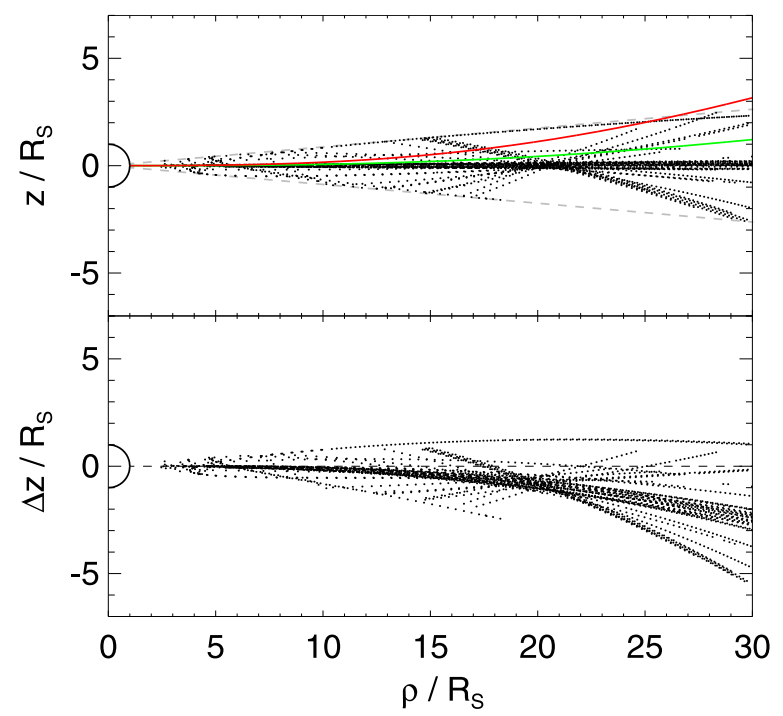

Figure 2. Plots showing the latitudinal coverage of the data during the interval of this study. The black points again show $1 \mathrm{~h}$ intervals containing magnetic data, as in Figure 1, but now plotted in cylindrical coordinates, where $z$ is directed along the planet's spin and magnetic axis and $\rho$ is the perpendicular distance from the axis. The solid lines show the position of the magnetic equator at the beginning (red) and end (green) of the interval according to the model of Arridge et al. [2008] (using a "hinging distance" of $29 \mathrm{R}_{\mathrm{S}}$ ). The $\pm 5^{\circ}$ latitude band employed in of this study is indicated by the grey dashed lines. The bottom plot then shows the axial displacement of the spacecraft, $\Delta z$, relative to the instantaneous modeled magnetic equator.

to October 2006), and 44-54 (May to December 2007), which provide coverage of the equatorial plane as shown in Figure 1. In this figure black points represent $1 \mathrm{~h}$ of magnetic field data obtained within $|\lambda| \leq 5^{\circ}$ plotted along the spacecraft trajectory, which is projected onto the planet's equatorial plane as viewed from the north, with the Sun to the right. The dashed lines are shown at $5 \mathrm{R}_{\mathrm{S}}$ intervals in radial distance and $2 \mathrm{~h}$ intervals in LT. The gray-scale coding according to the scale on the right indicates the total number of $1 \mathrm{~min}$ data points that fall into nonoverlapping spatial bins of $3 \mathrm{R}_{\mathrm{S}}$ in radial distance and $2 \mathrm{~h}$ in LT, as typically used in sections 3,5 , and 6 of this study. It can be seen that there is good data coverage in both radial distance and $\mathrm{LT}$ beyond $\sim 3 \mathrm{R}_{\mathrm{S}}$, with the notable exception of gaps in the afternoon sector (1400-1600 LT) at radial distances beyond $\sim 7 \mathrm{R}_{\mathrm{S}}$, and in the predawn sector $(0400-0600 \mathrm{LT})$ at radial distances of 6-12 $R_{S}$.

[12] The latitudinal coverage of the data employed in this study is shown in the top plot of Figure 2, where again the black points represent $1 \mathrm{~h}$ of magnetic field data plotted along the spacecraft trajectory, now shown in cylindrical $(\rho, z)$ coordinates. Approximately $\sim 70 \%$ of the data are confined to within $1^{\circ}$ of the planet's spin equator, while the remainder at large modular latitudes are distributed approximately equally $\sim 15 \%$ to the north and $\sim 15 \%$ to the south. We note, however that under the southern summer conditions prevailing during the interval of the study, the magnetic equatorial plane (where the radial field component reverses sign) is displaced consistently north of the planet's spin equator due to the component of the solar wind flow along the spin axis [e.g., Cowley et al., 2006]. The location of the magnetic equatorial plane as modeled by Arridge et al. [2008] is indicated in the figure by the red and green solid lines, corresponding to the start and end of this interval, respectively. It can be seen that the northward displacements are a fraction of an $R_{S}$ within the quasi-dipolar region, increasing to $\sim 2 R_{S}$ at the outer limit of the region studied here. It can also be seen that the magnetic equator is almost entirely contained within the $\pm 5^{\circ}$ range of latitudes employed, as indicated by the grey dashed lines in the figure. Thus the majority of the data were obtained within just a few $R_{S}$ of the magnetic equatorial plane. The bottom plot of Figure 2 shows the displacement of the spacecraft above or below the instantaneous modeled magnetic equator, the averaged modular value being $\sim 0.8 \mathrm{R}_{\mathrm{S}}$. Although these displacements are therefore typically very small, the northward offset of the magnetic equator is still sufficient to result in $\sim 88 \%$ of our data set being located south of the equator according to this model. Nevertheless this should not be a significant factor if the oscillatory perturbation fields are in phase either side of the magnetic equator, as suggested by the previous studies cited above.

\subsection{Analysis Procedure}

[13] As indicated in section 1, the analysis procedure employed here is based on the results of Provan et al. [2009a], whose study spans the whole of the interval we examine. Provan et al. [2009a] determined the oscillation phase of each magnetic field component within the core region during each periapsis pass of the spacecraft, by crosscorrelating suitably processed field data with a model rotating oscillation determined from the phase of the SKR modulation derived by Kurth et al. [2008], employed as an exact guide phase. As mentioned in section 1, the analysis showed that the oscillations in the $r$ and $\theta$ field components are generally in phase with each another, while those in the $\varphi$ field component are generally in lagging quadrature. They consequently modeled the field oscillations in the core region for field component " $i$ " as

$$
B_{i}=B_{i 0} \cos \left(\Psi_{M c}(\varphi, t)-\psi_{i}\right),
$$

where $\Psi_{M c}(\varphi, t)$ is the core magnetic phase function and the polarization of the oscillation is expressed as $\psi_{r}=\psi_{\theta}=$ $0^{\circ}$ and $\psi_{\varphi}=90^{\circ}$. The core phase function is expressed as

$$
\Psi_{M c}(\varphi, t)=\Phi_{M}(t)-\varphi,
$$

where $\varphi$ is the azimuthal angle (equivalent to LT) measured from noon positive toward dusk, thus corresponding to an $m=1$ oscillation as indicated in section 1 . The nearlinear magnetic phase function $\Phi_{M}(t)$ which defines the period of the field rotation is related to the corresponding function for the SKR modulation by

$$
\Phi_{M}(t)=\Phi_{S K R}(t)-\psi_{M}(t),
$$

where relative phase $\psi_{M}(t)$ is a function determined empirically from the cross-correlation fits to the periapsis pass data described above. Since both phase functions $\Phi_{M}$ and $\Phi_{S K R}$ depart only slightly from linear dependence on time $t$ during 
one oscillation, the period of the oscillations at any time is well approximated by

$$
\tau_{S K R}(t)=\frac{360^{\circ}}{\left(\frac{d \Phi_{S K R}(t)}{d t}\right)} \quad \text { and } \quad \tau_{M}(t)=\frac{360^{\circ}}{\left(\frac{d \Phi_{M}(t)}{d t}\right)},
$$

where the phase functions are expressed in degrees. The absolute value of $\Phi_{S K R}$ is defined such that maxima in radiated SKR power occur when

$$
\Phi_{S K R}(t)=360^{\circ} N
$$

for any integer value of $N$. Similarly we note that if $B_{r_{0}}=B_{\varphi_{0}}$ in equation (1), the resulting rotating uniform field in the core points in the direction $\varphi_{M}(t)$ relative to the Sun given by

$$
\varphi_{M}(t)=\Phi_{M}(t),
$$

again measured from noon positive toward dusk. The equatorial core field thus points in the sunward direction when $\Phi_{M}(t)=360^{\circ} \mathrm{N}$ for integer $\mathrm{N}$, and toward dusk for $\Phi_{M}(t)=360^{\circ} \mathrm{N}+90^{\circ}$ and so on, rotating in the same sense as the planet. The results of Andrews et al. [2008] and Provan et al. [2009a] then show that over the interval of simultaneous magnetic field and SKR phase data analyzed, the relative phase $\psi_{M}(t)$ is approximately constant (within the rather broad scatter of the SKR phase data), with an averaged value of $\sim 150^{\circ}$. Equations (5), (4) and (2b) then imply that at the time of SKR maxima, the direction of the equatorial core field is given by

$$
\varphi_{M} \approx 360^{\circ} N-150^{\circ} \approx 360^{\circ} N^{\prime}+210^{\circ},
$$

thus pointing radially outward at $\sim 0200 \mathrm{LT}$ as previously indicated in section 1 .

[14] We also note at this point that the SKR-based "SLS longitude" system discussed by Kurth et al. [2007, 2008] is defined in terms of the above parameters by

$$
\lambda_{S K R}(\varphi, t)=\Phi_{S K R}(t)-\varphi+100^{\circ},
$$

such that at a given instant $\lambda_{S K R}$ increases in the sense opposite to $\varphi$, and hence opposite to the sense of planetary rotation. It is thus directly related to our core region magnetic phase (from equation (2)) by

$$
\lambda_{S K R}(\varphi, t)=\Psi_{M c}(\varphi, t)+\psi_{M}(t)+100^{\circ} .
$$

Since the results of Andrews et al. [2008] and Provan et al. [2009a] have then shown that $\psi_{M}$ is approximately constant over the interval of simultaneous data, with an averaged value of $\sim 150^{\circ}$ as indicated above, we thus have

$$
\lambda_{S K R}(\varphi, t) \approx \Psi_{M c}(\varphi, t)+250^{\circ} .
$$

We may therefore approximately convert $\Psi_{M c}$ values to SLS longitude values by adding $250^{\circ}$ to $\Psi_{M c}$, or equivalently by subtracting $110^{\circ}$.

[15] The slowly-varying period of the SKR and magnetic field oscillations is then described by expressing the phase functions as polynomials of time $t$, specifically of fifth order for $\Phi_{S K R}(t)$ and third order for the relative phase $\psi_{M}(t)$.
Expressing $t$ in days since the beginning of 1 January 2004, the fifth order polynomial for $\Phi_{S K R}$ determined by [Kurth et al., 2008] from fits to SKR phase data over the interval 1 January 2004 to 10 August 2007 (corresponding to $0 \leq t \leq$ 1317 days) is given by

$$
\Phi_{S K R}(t)=\frac{360^{\circ}}{\tau_{0}} t-\Delta \Phi_{S K R}(t),
$$

where $\tau_{0}$ is precisely 0.4497 days $(10.793 \mathrm{~h})$, and

$$
\Delta \Phi_{S K R}(t)=k_{0}+k_{1} t+k_{2} t^{2}+k_{3} t^{3}+k_{4} t^{4}+k_{5} t^{5},
$$

where

$\begin{array}{llll}k_{0}=86.6681 & \text { deg } & k_{1}=-2.7537 & \text { deg day }^{-1} \\ k_{2}=4.7730 \times 10^{-3} & \text { deg day }^{-2} & k_{3}=-4.8755 \times 10^{-6} & \text { deg day }^{-3} \\ k_{4}=3.5653 \times 10^{-9} & \text { deg day }^{-4} & k_{5}=-9.1485 \times 10^{-13} & \text { deg day }^{-5}\end{array}$.

Similarly the magnetic phase function $\psi_{M}(t)$ determined by Provan et al. [2009a] is given by

$$
\psi_{M}(t)=k_{M 0}+k_{M 1} t+k_{M 2} t^{2}+k_{M 3} t^{3},
$$

where

$k_{M 0}=209.2 \quad \operatorname{deg} \quad k_{M 1}=-0.5718 \quad$ deg day $^{-1}$ $k_{M 2}=1.1446 \times 10^{-3}$ deg day $^{-2} k_{M 3}=-0.5995 \times 10^{-3}$ deg day $^{-3}$

Substitution into equation (3) then shows that the sidereal period of the magnetic field oscillations increased from $\sim 10.77 \mathrm{~h}$ at SOI in mid-2004 to $\sim 10.83 \mathrm{~h}$ by the end of 2007 , closely mirroring the corresponding variation in the SKR period over the interval from 2004 to August 2007 determined by Kurth et al. [2008].

[16] In this paper we use the Provan et al. [2009a] phase model to define the slowly varying period of the magnetic field oscillations in Saturn's magnetosphere during 20042007, thus allowing us to combine together the nearequatorial field data over this interval to study how the properties of the oscillations vary with radial distance and LT. Specifically we divide the field data (processed as described below) into various bins of radial distance and LT, and plot these binned values versus the core phase $\Psi_{M c}(\varphi, t)$ (modulo $360^{\circ}$ ). A sinusoid is then fitted to these data for each component $i$ using a simple least squares algorithm, thus yielding both the amplitude $B_{i_{0}}(r, \varphi)$ of the oscillation in that bin, and the phase $\psi_{i}^{*}(r, \varphi)$ with respect to the core phase $\Psi_{M c}(\varphi, t)$. The data in each bin is thus represented as

$$
B_{i}(r, \varphi, t)=B_{i 0}(r, \varphi) \cos \left(\Psi_{M i}(r, \varphi, t)\right)
$$

where

$$
\begin{aligned}
\Psi_{M i}(r, \varphi, t) & =\Psi_{M c}(\varphi, t)-\psi_{i}^{*}(r, \varphi) \\
& =\Phi_{M}(t)-\varphi-\psi_{i}^{*}(r, \varphi) .
\end{aligned}
$$

Clearly, given this procedure we expect to find $\psi_{r}^{*} \approx \psi_{\theta}^{*} \approx 0^{\circ}$ and $\psi_{\varphi}^{*} \approx 90^{\circ}$ within the core region inside $\sim 12 \mathrm{R}_{\mathrm{S}}$, in conformity with the previous analyses of Andrews et al. [2008] 


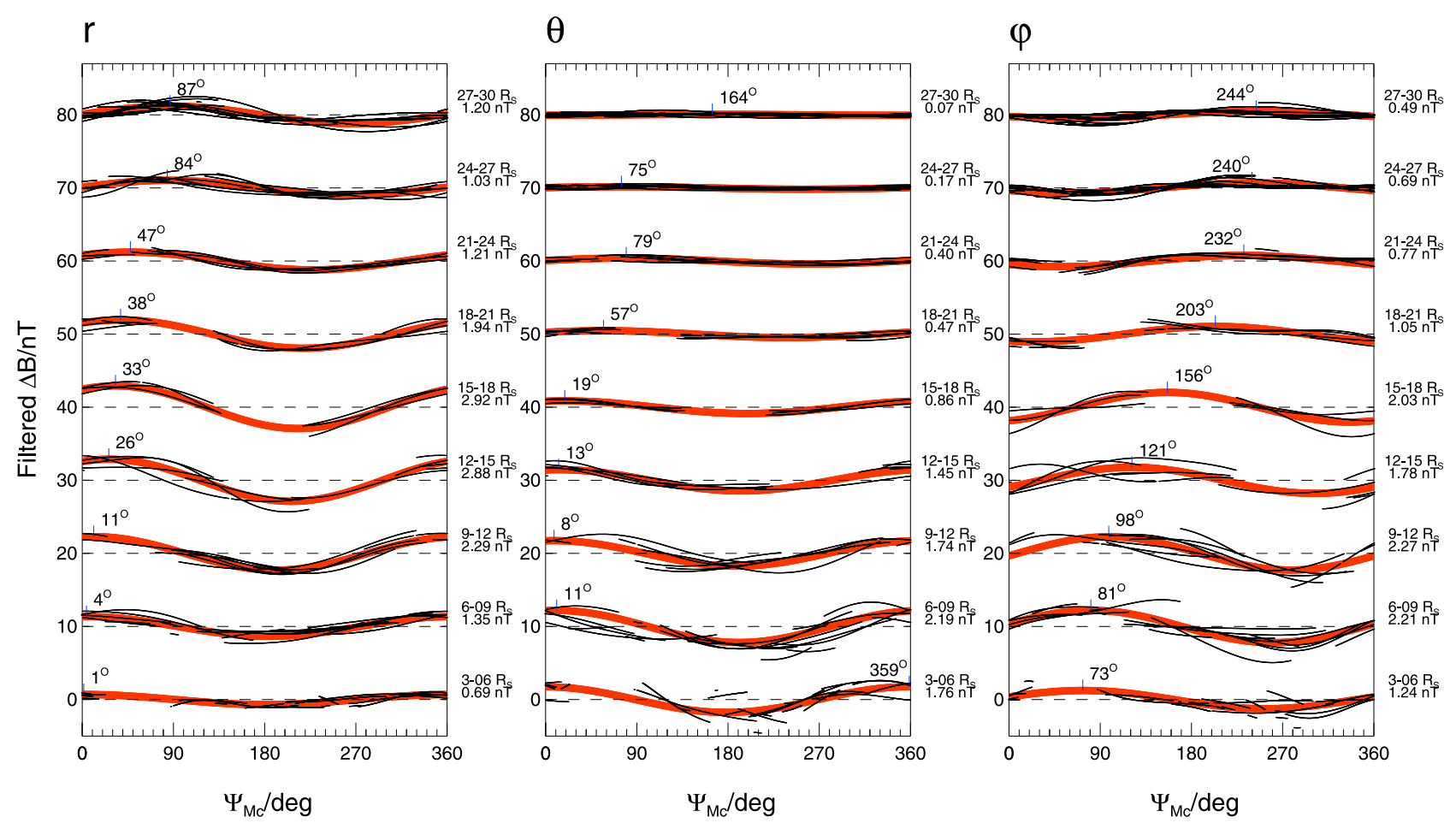

Figure 3. Plot showing processed magnetic field data (black lines) plotted versus the core phase, $\Psi_{M c}$, for each spherical polar component of the field ((left) $r$, (middle) $\theta$, and (right) $\varphi$ ), in a radial chain of 9 bins covering $2200-0200$ LT centered on local midnight. The bins are $3 R_{S}$ in radial width spanning the radial range $\sim 3-30 \mathrm{R}_{\mathrm{S}}$, and are stacked in the plot in order of increasing radial distance. The processed data consists of $1 \mathrm{~min}$ resolution band-pass filtered residual magnetometer data as described in section 2.2. The red traces show a sinusoidal fit to the data in each bin (equation (10)), obtained by a linear least squares method. The phase of the sinusoid, $\psi_{i}^{*}$, is indicated at its peak value in the plot for each bin, while the oscillation amplitude is indicated to the right of the data set to which it applies.

and Provan et al. [2009a], and equation (1). We note that this analysis method is similar to that previously employed by Gurnett et al. [2007] to determine the amplitude and phase of the oscillations in the $\varphi$ field component with radial distance using the SLS2 longitude system of Kurth et al. [2008]. Here, however, we extend the analysis to cover all three field components, and determine how the oscillation properties vary with LT as well as with radial distance.

[17] Prior to fitting equation (10) to the data in each spatial bin, we first process the magnetic field data by subtracting the planet's internal magnetic field using the "Cassini SOI" field model of Dougherty et al. [2005], leaving the residual field due to external sources. These data are then band-pass filtered using a Lanczos-squared filter with graduated cutoffs at periods of 5 and $20 \mathrm{~h}$ to extract the periodic effects potentially associated with the $\sim 11 \mathrm{~h} \mathrm{mag-}$ netospheric periodicity. The width of the filter band allows for the variable Doppler shifting that the oscillating signal undergoes due to the motion of the spacecraft [e.g., see Cowley et al., 2006]. In keeping with Provan et al. [2009a] we also exclude data from certain field components on certain orbits that contain significant field variations due to the planet's ring current that occur on comparable timescales to the magnetospheric period oscillations. On this basis we exclude $r$ and $\theta$ component data from Revs 29-33 and 41, and $r$ component data only from Revs 6-14, 34-36, 42, 46 and 54. We also exclude data in all cases that are obtained within $\pm 5 \mathrm{~h}$ of data gaps of more than $5 \mathrm{~h}$ duration, such that our filtered data are not significantly influenced by our practice of linearly interpolating over small $(<5 \mathrm{~h})$ data gaps. For this purpose, we note that data external to the magnetopause is treated as if it were a data gap.

[18] Finally, with regard to the size of the spatial bins employed in our analysis, we note that this necessarily involves a compromise between the amount of data in each bin and hence the accuracy of the resultant fit, and the spatial resolution of the amplitude and phase determinations. In practice the smallest bins we employ here (with hence the highest spatial resolution) are of $3 \mathrm{R}_{\mathrm{S}}$ in radial distance and $2 \mathrm{~h}$ in $\mathrm{LT}$, as employed in Figure 1.

\subsection{Sample Results}

[19] Figure 3 shows sample results from a radial chain of 9 bins centered on local midnight which in this case are of $4 \mathrm{~h}$ width in LT, such that each bin contains data from $2200-0200 \mathrm{LT}$. The radial width of each bin is $3 \mathrm{R}_{\mathrm{S}}$ as indicated above, covering intervals of $3-6,6-9, \ldots, 27-30 \mathrm{R}_{\mathrm{S}}$. Data for the $r, \theta$, and $\varphi$ field components are plotted separately in the three panels from left to right, with data in each panel stacked with increasing radial distance. In each bin, 
band-pass filtered residual magnetic field data are plotted as a function of the Provan et al. [2009a] "core phase" $\Psi_{M c}$, modulo $360^{\circ}$, shown by the black lines. The dashed lines indicate the zero values for each radial bin, with each successive bin being displaced upward by $10 \mathrm{nT}$. The red traces show the least squares fits of equation (10) to the data points in each bin. The amplitude of the fit in $\mathrm{nT}$ is indicated to the right of each plot along with the radial position of the bin, while the phase $\psi_{i}^{*}$ in degrees is indicated at the peak of the sinusoid. Data coverage of core phase $\Psi_{M c}$ is good throughout this region, such that the fitted parameters are well constrained by the data, though there are some bins (e.g., 15$18 \mathrm{R}_{\mathrm{S}}$ ) where phase gaps of $\sim 90^{\circ}$ are present. In general we place a limit of 330 on the minimum number of data points that must be present in a bin before amplitudes and phases are calculated, corresponding (with 1 min data) to $\sim 50 \%$ of an oscillation cycle, in an attempt to ensure a minimum reasonable phase coverage. However, each bin in Figure 3, typically contains $\sim 1000-5000$ points, so this criterion is well-satisfied in all bins in this case.

[20] It can readily be seen from Figure 3 that the oscillatory field in the inner 3-4 bins, corresponding to radial distances of $\sim 3-15 R_{S}$, is in good agreement with the results of previous studies, with oscillation phase values near zero for the $r$ and $\theta$ field components, and near $90^{\circ}$ for the $\varphi$ component, as expected. The phases are then seen to increase from these values with increasing radial distance, suggesting outward radial phase propagation as found previously by Gurnett et al. [2007] (in the $\varphi$ field component only), but by varying amounts in the three components of the field. The phase increase observed across the $\sim 27 R_{S}$ radial range covered here is $\sim 90^{\circ}$ in the $r$ and $\theta$ field components, but increases to $\sim 160^{\circ}$ in the $\varphi$ component, the implications of which will be discussed in in more detail in sections 3-6. Similarly, the amplitude is observed to increase with radial distance in each field component, before reaching a peak and then declining at larger radial ranges. Peak values of $\sim 2.2 \mathrm{nT}$ at $6-9 \mathrm{R}_{\mathrm{S}}$ are seen in the $\theta$ field component, $\sim 2.3 \mathrm{nT}$ at $9-12 \mathrm{R}_{\mathrm{S}}$ in the $\varphi$ field component, and $\sim 2.9 \mathrm{nT}$ at $15-18 \mathrm{R}_{\mathrm{S}}$ in the $r$ field component. In section 3 we survey corresponding results for all LT ranges, thus presenting an overall picture of the oscillating field in the equatorial region.

[21] First, however, we briefly discuss the uncertainties in our phase and amplitude determinations. The uncertainty in the phase is taken to be given by the change in the value required to yield a significant increase of $10 \%$ in the RMS deviation between the data and the best fit sinusoid away from the minimum value. The uncertainty in the amplitude is then taken to be given by the corresponding change between best fit values, and the mean of the values at the extrema of the above range of phases. The mean phase uncertainty of the data in Figure 3 estimated using this method is $\sim \pm 20^{\circ}$, comparable to the phase "jitter" in the data from individual passes [Andrews et al., 2008; Provan et al., 2009a], varying from a minimum of $\sim 7^{\circ}$ for the $r$ component in the range $15-18 \mathrm{R}_{\mathrm{S}}$, to a maximum of $\sim \pm 92^{\circ}$ in the $\theta$ component at $27-30 \mathrm{R}_{\mathrm{S}}$. Analysis shows that, in general, the phase uncertainty grows approximately linearly with the ratio of the RMS deviation over the best fit amplitude, with the uncertainty increasing to $\sim \pm 90^{\circ}$ when the latter ratio increases to $\sim 1.5$. In sections $3-6$, therefore, values are not shown when the RMS deviation exceeds this limit. The uncertainty in the amplitude values in Figure 3 is found to be $\sim \pm 10 \%$, varying between $\sim \pm 2 \%$ for the $r$ component in the range $12-15 \mathrm{R}_{\mathrm{S}}$ and $\sim \pm 90 \%$ in the $\theta$ component in the range $27-30 \mathrm{R}_{\mathrm{S}}$. Application of the above criterion also leads to the removal of data with such large amplitude uncertainties, approaching $\pm 100 \%$. In practice, the criterion leads to the exclusion of results from a small number bins where the number of field measurements is low (though exceeding the initial limit of 330), the phase coverage poor, or the amplitude of the oscillation is very small. In Figure 3, for example, only the results from the outermost bin of the $\theta$ field component are excluded, in which the fitted amplitude is $\sim 0.07 \mathrm{nT}$, a factor of $\sim 3$ smaller than the RMS deviation of the data about that fit. The phase determined from the fit in this bin is indeed notably discrepant relative to the phases in adjacent bins.

\section{Amplitude and Phase of the Field Oscillations in the Equatorial Region}

\subsection{Variation With Radial Distance and LT}

[22] Overall results are shown in Figure 4. In Figure 4a we plot the amplitude and phase of the oscillations versus radial distance in fixed LT sectors, while in Figure $4 \mathrm{~b}$ we plot these quantities versus LT at fixed radial distances in a complementary format. Specifically, in Figure 4a we use bins of $3 R_{S}$ in radial distance (i.e., 3-6, 6-9, .., 27-30 $R_{S}$ ) and $4 \mathrm{~h}$ bins in LT (as in Figure 3), where the color coding indicates the different LT sectors as indicated in the diagram in the top left corner of the figure (which has a format similar to Figure 1). Thus, for example, the black traces in Figure $4 \mathrm{a}$ correspond to the $4 \mathrm{~h}$ LT sector centered on midnight (2200-0200 LT), as shown previously in Figure 3. Similarly, in Figure $4 \mathrm{~b}$ we employ bins of $2 \mathrm{~h}$ in LT and $6 \mathrm{R}_{\mathrm{S}}$ in radial distance $\left(3-6,6-12, \ldots, 24-30 \mathrm{R}_{\mathrm{S}}\right)$, where the plots are now color coded according to radial range as again indicated in the diagram in the top left corner. The plots in the top right corner of each figure indicate the number of 1 min data points, $N$, in each bin, for each field component. The bottom plots in these figures then show from left to right, the amplitudes and phases of the oscillations in the $r$, $\theta$, and $\varphi$ field components, respectively.

[23] We begin our discussion with the oscillation in the $\theta$ field component (directed North-South through the equatorial plane), the amplitudes and phases of which are plotted in the bottom middle plots of Figures $4 \mathrm{a}$ and $4 \mathrm{~b}$. The radial profiles of amplitude in Figure 4 a show a peak at 6-9 $R_{S}$, falling modestly by $\sim 0.5 \mathrm{nT}$ in the inner $3-6 \mathrm{R}_{\mathrm{S}}$ range, and near-monotonically to small values at larger radial distances, particularly beyond $\sim 15 \mathrm{R}_{\mathrm{S}}$. The peak values themselves are somewhat larger on the nightside, $\sim 1.5-2 \mathrm{nT}$, than on the dayside, $\sim 1-1.5 \mathrm{nT}$, though the overall variation with LT is not large as can be seen explicitly in Figure $4 \mathrm{~b}$. Turning to the phase values shown in the bottom middle plots it can be seen that values are close to zero throughout the core, as expected, but rise slowly with increasing radial distance, suggestive of an outward propagating disturbance. Phase delays with increasing radial distance are larger on the nightside than on the dayside, reaching $\sim 90^{\circ}$ in the postdusk and midnight sectors at the outer edge of the region examined, such that the oscillations in this sector at these dis- 


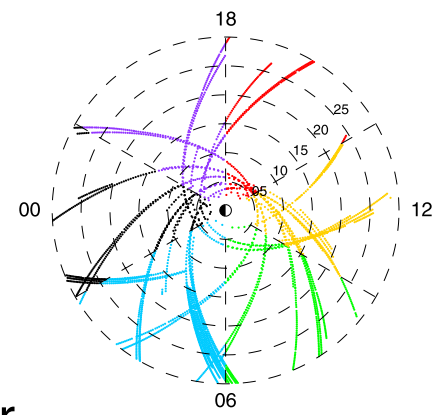

r

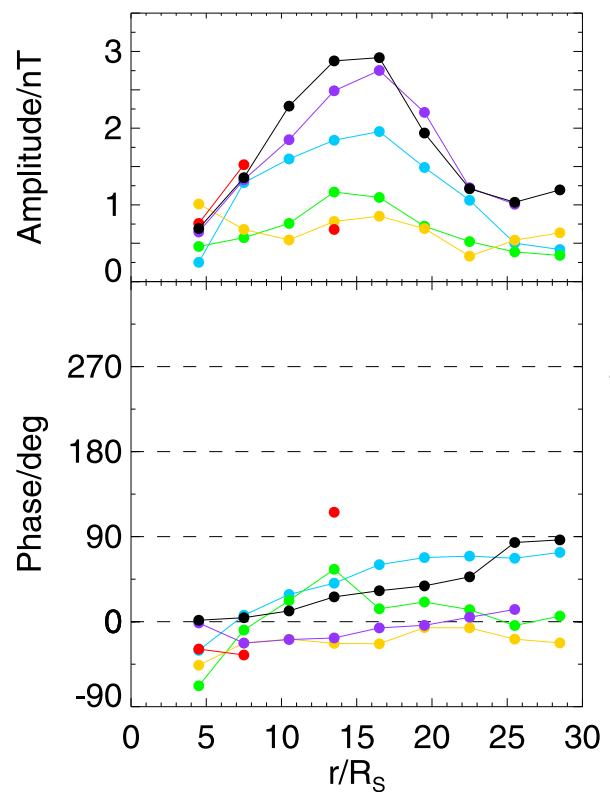

$\theta$

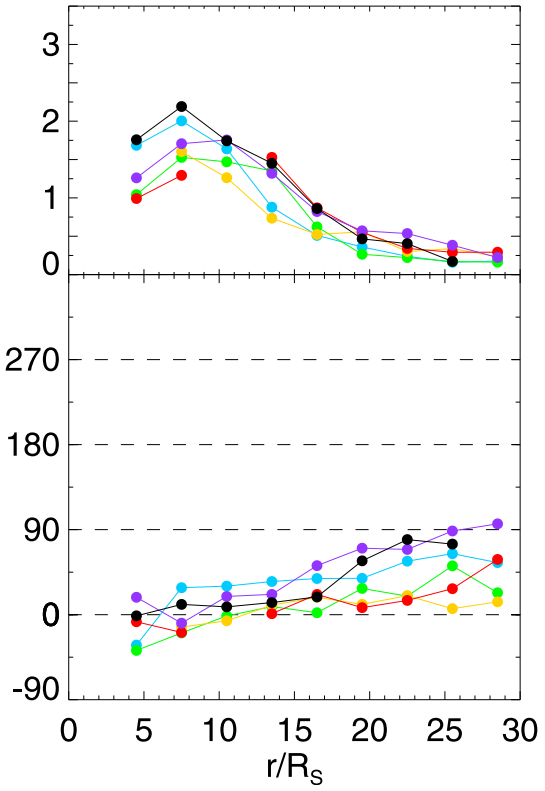

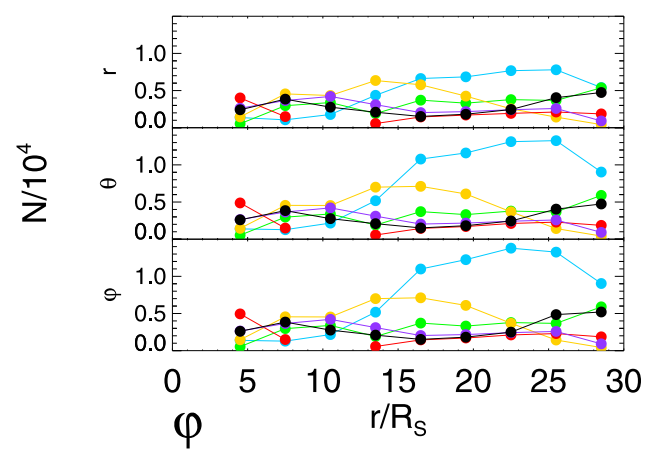

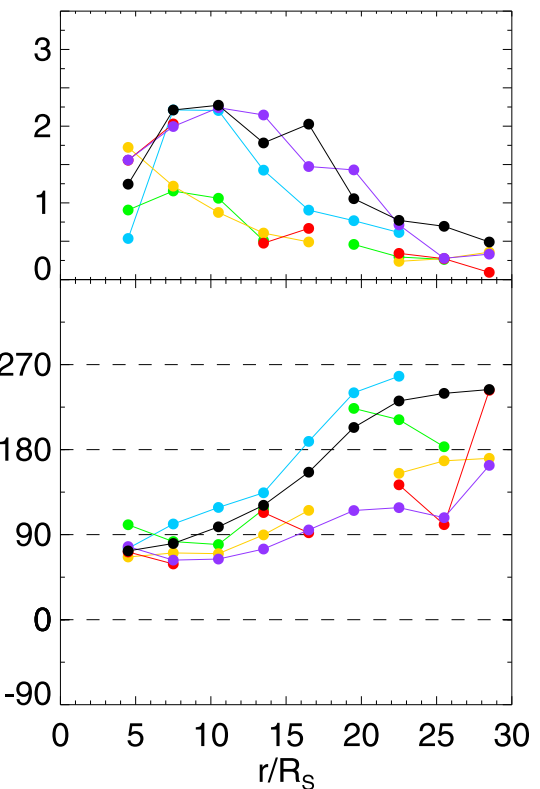

Figure 4a. Plots of the oscillation amplitude and phase versus radial distance, using bins of $3 \mathrm{R}_{\mathrm{S}}$ in radial distance and $4 \mathrm{~h}$ in LT, with the color coding indicating the LT sector of each bin, as depicted in the diagram in the top left corner of the figure (which has a format similar to Figure 1). The number of $1 \mathrm{~min}$ data points in each bin is indicated in the plots shown in the top right corner of the figure, for each field component. The bottom plots indicate the amplitude and phase of the oscillation, respectively, determined as outlined in section 2, for each field component (left) $r$, (middle) $\theta$, and (right) $\varphi$.

tances are in approximate lagging quadrature with respect to those in the core, as previously noted in the discussion of Figure 3. Phase variations with radius are generally significantly smaller on the dayside, with almost no phase change with radial distance occurring in the noon sector. These results thus suggest that the effective radial phase speed is significantly larger on the dayside than on the nightside, as will be quantified in section 4 .

[24] We now examine the oscillations in the $r$ and $\varphi$ field components (lying in the equatorial plane), results for which are shown in the bottom left and right plots of Figures $4 \mathrm{a}$ and $4 \mathrm{~b}$. The amplitude variations in these two field components show generally similar behavior, rising from small values in the inner region to peak at larger distances, before falling to small values at the outer edge of the region considered. However, there is a much stronger dependence on LT evident in these components than in the $\theta$ component, with peak amplitudes typically maximizing in the premidnight sector ( 2000-2400 h LT) as can be seen in both Figures $4 \mathrm{a}$ and $4 \mathrm{~b}$. Indeed, it can be seen that in the noon sector (orange traces in Figure 4a) no significant peak in amplitude is present at all, the amplitudes being small and approximately constant with radius in the $r$ field component, and monotonically falling with increasing radius in the $\varphi$ component.

[25] In more detail, the amplitude of the $\varphi$ field component is $\sim 1-1.5 \mathrm{nT}$ in the inner region at 3-6 $\mathrm{R}_{\mathrm{S}}$ (comparable to that in the $\theta$ component), peaks on the nightside at $\sim 2 \mathrm{nT}$ in the radial range $6-15 \mathrm{R}_{\mathrm{S}}$ corresponding to the outer core region, and then generally falls with radial distance with values on the nightside typically larger than that of the $\theta$ component beyond $\sim 9 \mathrm{R}_{\mathrm{S}}$. The amplitude of the $r$ field component, however, is significantly smaller than that of the $\varphi$ and $\theta$ components in the inner region, with values of $\sim 0.5-1 \mathrm{nT}$, while growing to larger peak values of $\sim 2-3 \mathrm{nT}$ on the nightside at larger radial distances of $\sim 15-18 \mathrm{R}_{\mathrm{S}}$. The nightside amplitudes of the $r$ field component then remain larger than those of both the $\varphi$ and $\theta$ components as they again fall with increasing radial distance. Overall, therefore, the oscillations in the equatorial field components are 

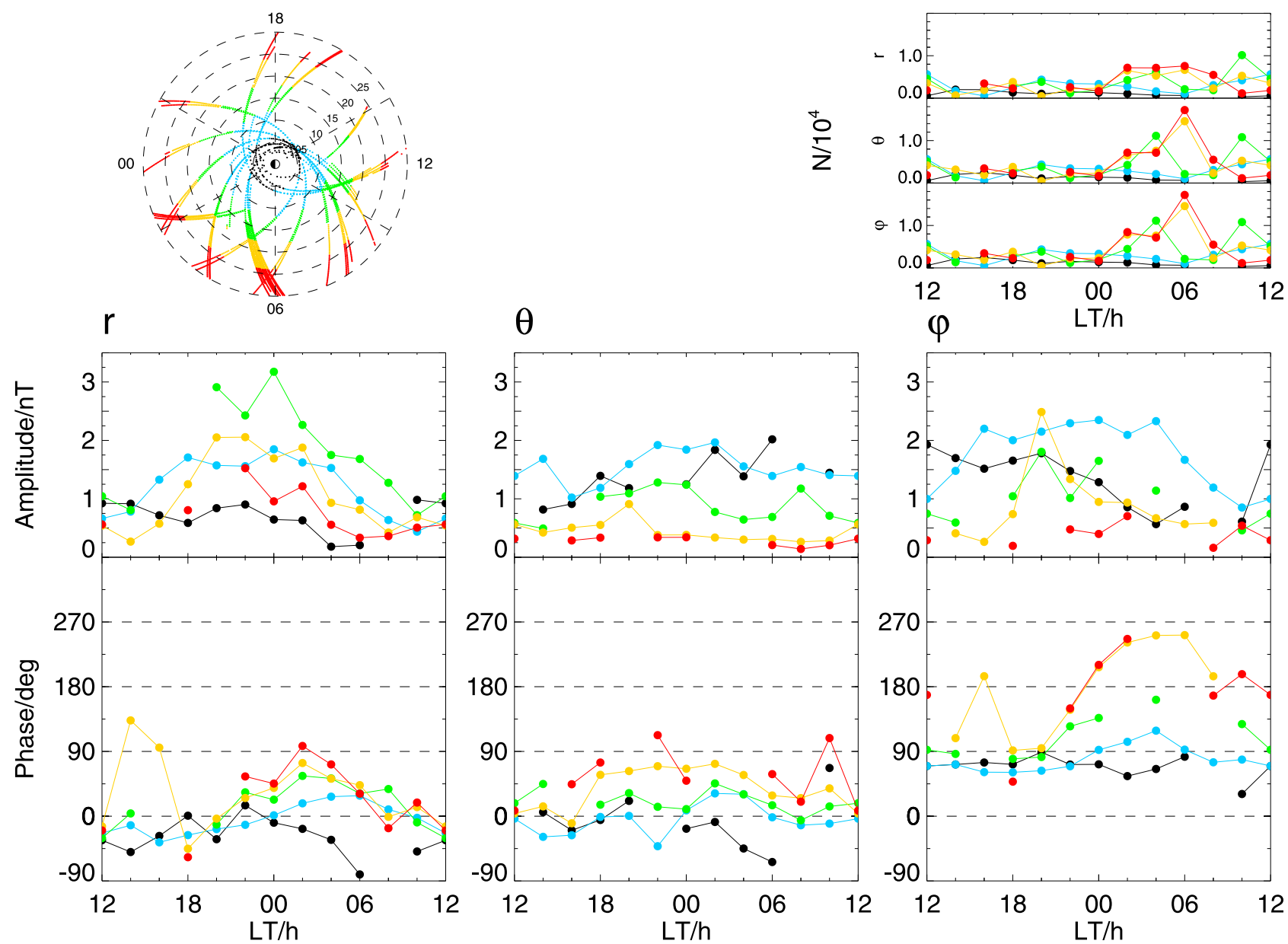

Figure 4b. Similar to Figure 4a, but now with the amplitude and phase of the oscillation plotted versus LT using $6 \mathrm{R}_{\mathrm{S}}$ bins of radial distance and $2 \mathrm{~h}$ in LT, with the color coding indicating radial distance as depicted in the diagram in the top left corner.

dominated by the $\varphi$ component in the inner region between 3 and $9 \mathrm{R}_{\mathrm{S}}$, and by the $r$ component beyond $\sim 12 \mathrm{R}_{\mathrm{S}}$. The significance of this result for the form of the oscillating field will be discussed further in section 5 .

[26] Examining now the phase values of the $r$ and $\varphi$ field components, it can be seen that the phase behavior of the $r$ component is generally similar to that of the $\theta$ component, with near-zero values in the inner region, increasing slowly with radial distance, more rapidly on the nightside than on the dayside. However, a significant departure is observed in the postdusk sector (purple trace in Figure 4a), where the phase of the $r$ field component increases by only a small amount between the inner and outer regions, similar to the behavior on the dayside, while the phase of the $\theta$ component increases by $\sim 90^{\circ}$ in the same sector. Thus while our results indicate that these field components generally oscillate in phase throughout the equatorial region, the $r$ component moves into approximate leading quadrature with the $\theta$ component in the postdusk sector. The behavior of the $\varphi$ field component is somewhat more complex. In the inner region, out to $\sim 15 \mathrm{R}_{\mathrm{S}}$, the phase values are near $\sim 90^{\circ}$ as expected, increasing gradually with radial distance in a manner similar to that of the $r$ and $\theta$ components, such that $\varphi$ remains in approximate lagging quadrature with $r$ and $\theta$ throughout this region. However, the $\varphi$ component phase increases much more rapidly across $15-21 \mathrm{R}_{\mathrm{S}}$, by $\sim 90^{\circ}$ in the midnight and dawn sector (black, blue, and green traces in Figure 4a) and by lesser amounts at noon and dusk (orange, red, and purple traces), before leveling out once more in the outer region. The net result is that while the $\varphi$ field component remains in lagging quadrature with the $r$ component to large distances in the dusk sector, it moves toward antiphase at other LTs, and even toward leading quadrature with $r$ in a region near dawn.

\subsection{Phase Fronts and Phase Differences}

[27] A more graphic representation of the above phase data is presented in Figure 5, where we show color coded plots of the full oscillation phase function (given by equation (10b), i.e., $\Psi_{M i}=\Phi_{M}-\varphi-\psi_{i}^{*}$ (modulo $360^{\circ}$ ) for field component $i$, versus radial distance and LT in the equatorial plane, at two points in the oscillation cycle. Plots are shown for the $r, \theta$, and $\varphi$ field components from left to right, as in Figure 4, where the top row of plots corresponds 
(a) r-component

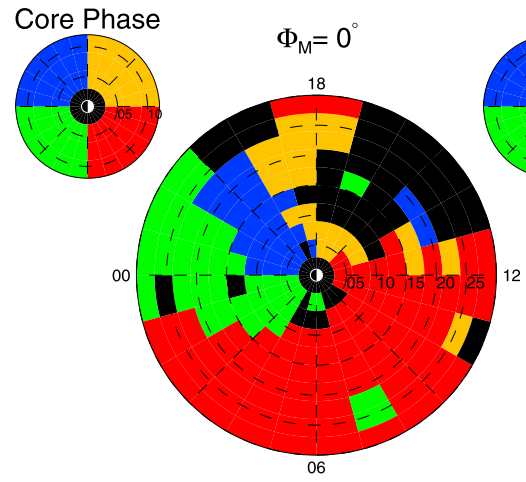

(b) $\theta$-component

(c) $\varphi$-component

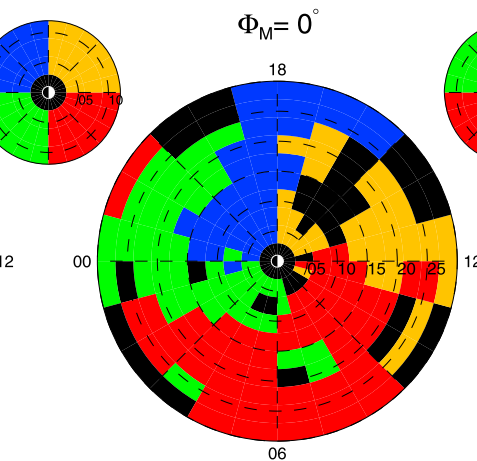

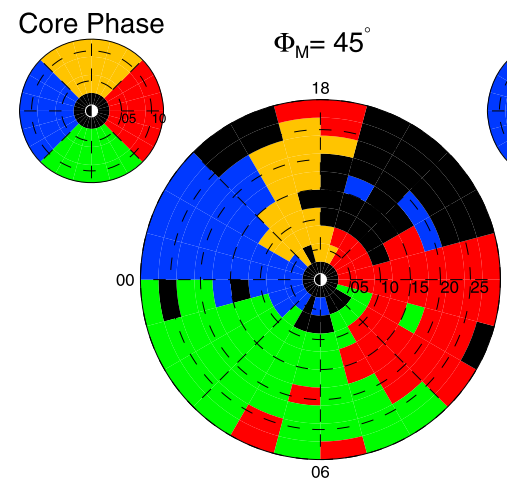
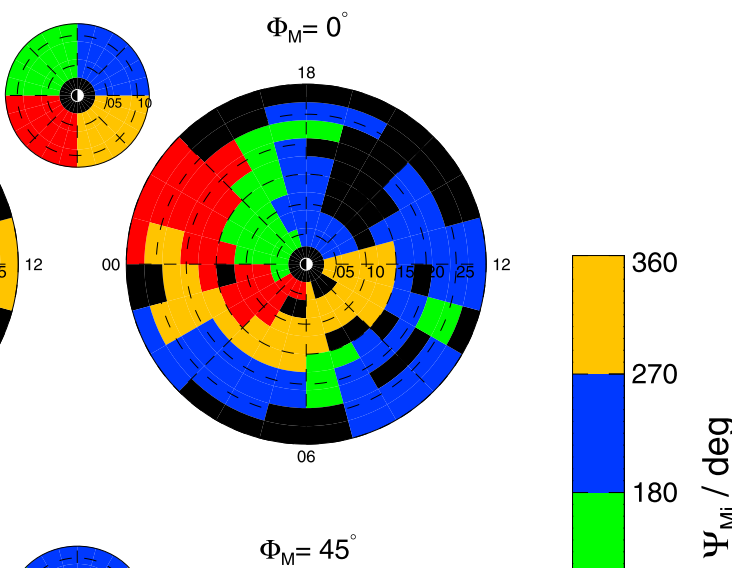

Figure 5. Plots showing the oscillation phase $\Psi_{M i}$ for each magnetic field component, given by equation (10), at two instances of $\Phi_{M}$, plotted versus radial distance and LT on the equatorial plane, determined using bins of $3 \mathrm{R}_{\mathrm{S}}$ in radial distance and $2 \mathrm{~h}$ in LT, computed every hour. The phase is color coded in $90^{\circ}$ increments as indicated by the color bar on the right of the figure, such that the field in any component is positive in the red and orange sectors, and negative in the blue and green. The format is otherwise similar to Figure 1. The inset icon in the top left corner of each plot shows the expected core phase out to $\sim 12 \mathrm{R}_{\mathrm{S}}$ as determined by Provan et al. [2009a], given by equation (2a). Results for the (a) $r$, (b) $\theta$, and (c) $\varphi$ field components are shown, where the top row of plots corresponds to $\Phi_{M}=0^{\circ}$, and the bottom row to $\Phi_{M}=45^{\circ}$.

to $\Phi_{M}=0^{\circ}$, and the bottom row to $\Phi_{M}=45^{\circ}$. The color scale is shown on the right, and is such that field components are positive in the red and orange regions, with $\cos \Psi_{M i}=1$ at their interface, and negative in the green and blue regions, with $\cos \Psi_{M i}=-1$ at their interface. Correspondingly, zeros in the field are present at the interfaces between the red and green, and the blue and orange regions. The inset icons in the top left of each plot indicate the expected results within the core region if the phase exactly obeys the model derived by Provan et al. [2009a], given by equation (1). For example, at $\Phi_{M}=0^{\circ}$ when the equatorial core field points toward the Sun (equation (5)), the core phase is zero on the noon meridian, and increases clockwise around the plot such that the $r$ and $\theta$ field components are positive on the dayside and negative on the nightside. For the $\varphi$ field component at $\Phi_{M}=$ $0^{\circ}$ this pattern is rotated clockwise through $90^{\circ}$, such that the field is positive at dawn and negative at dusk. Similarly when $\Phi_{M}=45^{\circ}$ in the bottom row, the equatorial core field points $45^{\circ}$ duskward, and each core phase pattern is rotated anticlockwise by $45^{\circ}$.

[28] We now compare these model core phases with the phase plots shown, which have been determined using radial bins of $3 R_{S}$ as before, but now with $2 \mathrm{~h}$ bins in LT com- puted every hour (such that adjacent bins overlap by $0.5 \mathrm{~h} \mathrm{LT}$ ). Beginning as before with results for the $\theta$ field component shown in the central column, it can be seen that the phase is largely in agreement with the core model out to $\sim 15 \mathrm{R}_{\mathrm{S}}$ as expected, but that beyond this the nightside phase fronts become increasingly "swept back" to earlier LT with increasing radius such that the phase in the outer nightside region is approximately in lagging quadrature with the core, as indicated in section 3.1. However, the effect on the dayside is much less marked. As a consequence of this dependency on LT, the phase fronts become compressed together in the dusk sector, and expanded away from each other in the dawn sector. Thus, for example, at larger radii the "blue" region at $\Phi_{M}=0^{\circ}$ is generally confined to a narrow region in the postdusk sector, while the "red" region extends from noon all the way through the postmidnight sector. The effect of this on the azimuthal propagation of the oscillation becomes evident on comparing the plots for $\Phi_{M}=$ $0^{\circ}$ and $\Phi_{M}=45^{\circ}$. Here the "orange" phase region becomes compressed in the outer region within the dusk sector, while the "green" phase region expands rapidly through the dawn sector, confining the red sector largely within the noon quadrant. Thus, in the outer region the azimuthal phase 
propagation is retarded at dusk and enhanced at dawn, as will be quantified in section 4. Further evolution of this phase pattern with increasing $\Phi_{M}$ can readily be envisaged from the plots shown in Figure 5 through transformation of the colors. For example, at $\Phi_{M}=90^{\circ}$ the blue areas in the $\Phi_{M}=$ $0^{\circ}$ plots transform into green, the green into red and so on. Alternatively, animations of the propagating phase fronts may be viewed in the auxiliary online material in Animations S1$\mathrm{S} 3$ for each of the three field components. ${ }^{1}$

[29] Turning now to the phase of the $r$ field component shown on Figure 5a, it can be seen that the behavior is similar to the $\theta$ component as expected from the largely in-phase behavior of these components. However, the phase fronts postdusk are now advanced compared with the core region, rather than being swept back, such that $r$ is in leading quadrature with $\theta$ in this sector as noted in section 3.1. As a consequence, the phase fronts in the outer region are even more compressed in this sector than for the $\theta$ field component, with the result that at $\Phi_{M}=0^{\circ}$ negative radial field values are confined to a narrow $\sim 6 \mathrm{~h}$ range of LT between $\sim 20$ and $02 \mathrm{~h}$ (blue and green regions) while positive values are found over the remaining $\sim 18 \mathrm{~h}$ of LT (red and orange regions). Of course, these polarities reverse sense at $\Phi_{M}=$ $180^{\circ}$. Again, this implies azimuthal phase propagation that is retarded in the dusk sector and enhanced in the dawn sector for this field component.

[30] Results for the $\varphi$ field component on Figure 5c again show good agreement with the core phase within $\sim 15 \mathrm{R}_{\mathrm{S}}$, as anticipated from the discussion in section 3.1. However, at LTs from midnight to dawn and noon, much stronger sweep back of the phase fronts occurs in the radial range just beyond this region, such that the phase in the outer region is in approximate lagging quadrature with those of the core at the same LT. An exception to this occurs in the dusk sector where the phase does not vary greatly with radial distance, except toward the edge of the region considered.

[31] The implications of these results for the phase difference between the three field components, and thus the overall structure of the oscillatory field, are quantified in Figure 6. Here, the left column of plots examines the phase difference between the $r$ and $\theta$ field components, the middle column the phase difference between the $\varphi$ and $\theta$ components, and the right column the phase difference between the $\varphi$ and $r$ components. The labels at the top of the plots " $i-j$, for field components $i$ and $j$ thus indicate that the phase differences plotted beneath correspond to $\Delta \psi^{*}=\psi_{i}^{*}-\psi_{j}^{*}$. Figure 6a then shows the phase difference versus radial distance color coded according to LT in the same format as Figure 4a, Figure 6b shows these values versus LT color coded according to radial distance in the same format as Figure $4 b$, while Figure $6 c$ shows the color coded phase difference in a format similar to Figure 5. However, the color scale of the latter, shown on the right, has been revised relative to the Figure 5, such that red corresponds to the $\pm 45^{\circ}$ band of phase centered on $0^{\circ}$, green to the $\pm 45^{\circ}$ band centered on $90^{\circ}$, an so on. Red regions thus indicate that field component $i$ is approximately in phase with component $j$, while blue indicates antiphase. Green indicates $i$ is in lagging quadrature with $j$ and orange vice versa.

\footnotetext{
${ }^{1}$ Auxiliary materials are available in the HTML. doi:10.1029/ 2009JA014729.
}

[32] Examining first the $r-\theta$ results in the left-hand column, it can be seen that these field components are approximately in phase not only in the core region but also throughout the dawn-side magnetosphere (red region in the bottom plot). However, the $r$ field component moves into leading quadrature with $\theta$ (orange region) at larger distances in the postdusk sector as indicated above, while there is also some indication of $\theta$ being in leading quadrature with $r$ (blue regions) at similar distances in the predusk sector, within the limited spatial coverage of that data. Similarly, the $\varphi-r$ results in the right column show that $\varphi$ is in lagging quadrature with $r$ throughout the core region, extending into the outer dusk sector (green region in the bottom plot). However, $\varphi$ is approximately in antiphase with $r$ in the outer region at the other LTs (blue), extending from midnight through dawn to noon, with the phase difference increasing such that $\varphi$ becomes approximately in leading quadrature with $r$ in the outer region near the dawn meridian (orange region). The $\varphi-\theta$ plots in the middle column of Figure 6 are then essentially similar to the $\varphi-r$ plots on the right, as expected from the mainly in phase $r-\theta$ relationship. However, the changing phase between $r$ and $\theta$ in the outer postdusk sector leads to $\varphi$ and $\theta$ becoming approximately in phase in that region. The consequences of these relationships for the overall structure of the oscillatory field in the equatorial plane will be discussed in section 5 .

\section{Radial and Azimuthal Phase Speeds}

[33] In section 3 it was noted that the generally increasing values of the oscillation phase with radial distance implies outward radial propagation of the phase fronts with a speed that is generally larger on the dayside than on the nightside. The related LT dependence of the sweep back of the phase fronts similarly suggests slower azimuthal propagation at dusk than at dawn. In this section we quantify these effects by calculating the radial and azimuthal phase velocities explicitly from the phase data.

[34] The rate of change of the phase $\Psi_{M i}=\Phi_{M}(t)-\varphi-$ $\psi_{i}^{*}(r, \varphi)$ (equation $\left.(10 \mathrm{~b})\right)$ for a point $(r, \varphi)$ on the equatorial plane moving with velocity $\left(v_{r}, v_{\varphi}\right)$ is given by

$$
\frac{d \Psi_{M i}}{d t}=\frac{\partial \Psi_{M i}}{\partial t}+\vec{v} \cdot \vec{\nabla} \Psi_{M i}=\Omega_{M}-v_{r} \frac{\partial \psi_{i}^{*}}{\partial r}-\frac{v_{\varphi}}{r}\left(1+\frac{\partial \psi_{i}^{*}}{\partial \varphi}\right)
$$

where

$$
\Omega_{M}=\frac{d \Phi_{M}}{d t}
$$

is the instantaneous angular frequency of the core phase model of Provan et al. [2009a], obtained by differentiation of equation (10b) (using equations (8) and (9)). We then obtain an expression for the radial phase speed by setting $v_{\varphi}=0$, and determining the speed $v_{r}$ so that $d \Psi_{M i} / d t=0$, such that we move exactly radially with a point of given phase $\Psi_{M i}$. The radial phase speed is thus given by

$$
v_{r i}=\frac{\Omega_{M}}{\left(\frac{\partial \psi_{i}^{*}}{\partial r}\right)} .
$$



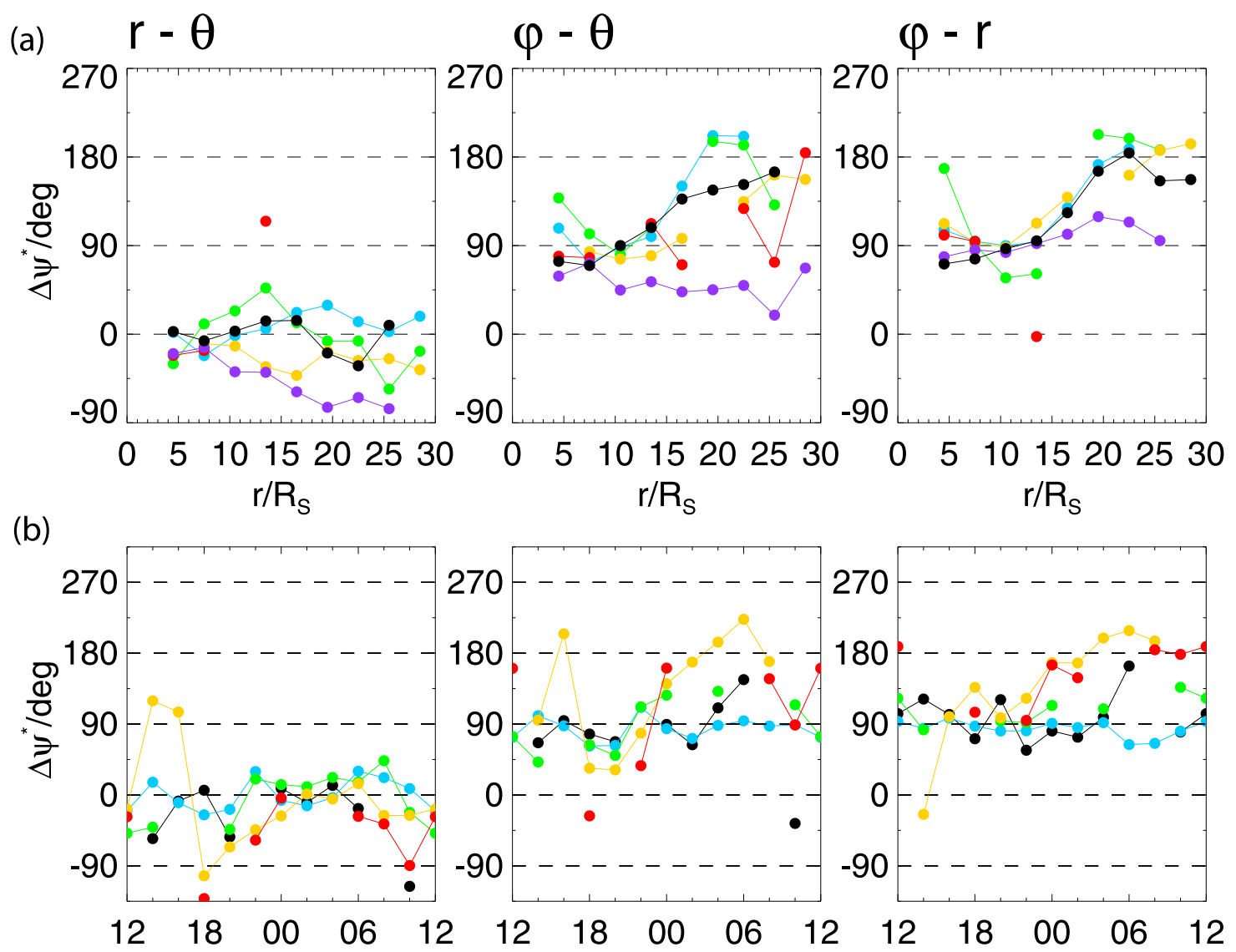

$\mathrm{LT} / \mathrm{h}$

(c)
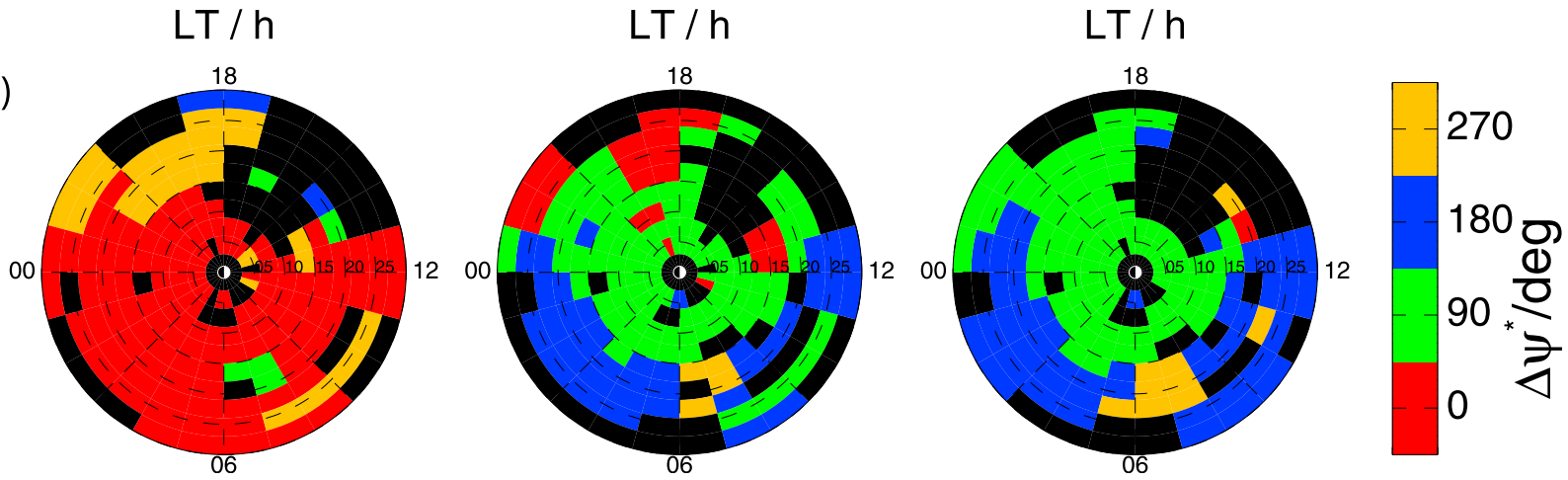

Figure 6. Plots showing the phase differences between each pair of field components, (left) $\psi_{r}^{*}-\psi_{\theta}^{*}$, (middle) $\psi_{\varphi}^{*}-\psi_{\theta}^{*}$, and (right) $\psi_{\varphi}^{*}-\psi_{r}^{*}$, plotted versus (a) radial distance, (b) LT, and (c) projected onto the equatorial plane. From Figures $6 \mathrm{a}, 6 \mathrm{~b}$, and $6 \mathrm{c}$ the differences plotted in each row thus correspond to the differences in the individual component phase data presented in Figures $4 \mathrm{a}, 4 \mathrm{~b}$, and 5, respectively. The color coding in Figure $6 \mathrm{a}$ thus indicates LT using the same format as Figure 4a, while that in Figure $6 \mathrm{~b}$ indicates radial distance in the same format as Figure $4 \mathrm{~b}$. The color code employed in Figure $6 \mathrm{c}$, shown in the color bar on the right, is such that the two components are approximately in phase $\left( \pm 45^{\circ}\right)$ in the red regions and antiphase in the blue regions, while the first component (e.g., $r$ in the left column) is in lagging quadrature with the second ( $\theta$ in the left column) in the green regions, and vice versa in the orange regions.

We note that $\Omega_{M}$ in the above expression is not quite constant, but varies over the interval of this study by $\sim 1 \%$ as discussed in section 2 . However, since this variation is very small, for simplicity we here employ a fixed representative value taken to be $\Omega_{M}=0.16160 \times 10^{-3} \mathrm{rad} \mathrm{s}^{-1}$, equivalent to a rotation period of $10.800 \mathrm{~h}$, thus corresponding specifically to $t \approx 650 \mathrm{~d}$, near the center of the interval considered (12 October 2005). Similarly, setting $v_{r}=0$ in equation (11a), 
we obtain an expression for the azimuthal phase velocity, normalized to $\Omega_{M}$, as

$$
\frac{\Omega_{i}}{\Omega_{M}}=\frac{1}{\left(1+\frac{\partial \psi_{i}^{*}}{\partial \varphi}\right)} .
$$

[35] Considering first the radial phase speed given by equation (12a), we reproduce the phase values $\psi_{i}^{*}$ versus radial distance in the top plots of Figure $7 \mathrm{a}$, as shown previously in Figure $4 a$, determined in bins of $3 R_{S}$ in radial distance and in six color coded $4 \mathrm{~h}$ bins in LT. To evaluate the radial phase speed from equation (12a) we first need to determine the radial gradient of $\psi_{i}^{*}$, and given the nature of these data only an overall linear fit seems appropriate (piecewise for the $\varphi$ field component as discussed below). These fits are shown by the similarly color coded solid lines in Figure 7a, the gradients of which yield the overall phase speed in each LT sector, for each field component oscillation, as plotted in the bottom plots of Figure 7a. The error bars shown are determined from the $1-\sigma$ uncertainties in the gradients of the linear fits.

[36] Beginning with results for the $\theta$ field component shown in the middle plots of Figure 7a, it can be seen that the phase speeds in the midnight and premidnight sectors are typically $\sim 150 \mathrm{~km} \mathrm{~s}^{-1}$, increasing to $\sim 200 \mathrm{~km} \mathrm{~s}^{-1}$ in the predusk and dawn sectors, though with larger error bars. The estimated phase speed peaks in the noon sector at $\sim 500 \mathrm{~km}$ $\mathrm{s}^{-1}$, with substantially larger error bars. Similar, but more scattered results are obtained for the $r$ field component, as shown on the left of Figure 7a, with values of $\sim 150 \mathrm{~km} \mathrm{~s}^{-1}$ in the midnight and predawn sectors, increasing to $\sim 400$ $600 \mathrm{~km} \mathrm{~s}^{-1}$ postdusk and postdawn and to $\sim 700 \mathrm{~km} \mathrm{~s}^{-1}$ in the noon sector, the latter again with large error bars.

[37] Results for the $\varphi$ component have been treated somewhat differently due to the presence of the rapid phase increase near $\sim 15 \mathrm{R}_{\mathrm{S}}$ associated with the spatial structure of the oscillatory field, as will be discussed in section 5 . Consequently, we have made separate linear fits to the data in the inner region (3-15 $R_{S}$ ) shown by solid circles, and to the data in the outer region $\left(18-30 \mathrm{R}_{\mathrm{S}}\right)$ shown by solid triangles, omitting data from $15-18 \mathrm{R}_{\mathrm{S}}$ shown by the open circles. Corresponding radial phase speeds are shown by the solid circles and triangles in the lower plot, with offset error bars for clarity. Phase speeds are again $\sim 100-150 \mathrm{~km} \mathrm{~s}^{-1}$ on the nightside, increasing to $\sim 250-350 \mathrm{~km} \mathrm{~s}^{-1}$ on the dayside, though with larger error bars. There are also some radial ranges and LT sectors where the phase speed is apparently negative, indicating inward motion of the phase fronts.

[38] Results for the normalized angular velocity associated with azimuthal phase propagation are similarly shown in Figure 7b. The top plots again reproduce the phase values $\psi_{i}^{*}$ as plotted previously in Figure $4 \mathrm{~b}$ (using bins of $6 \mathrm{R}_{\mathrm{S}}$ in radial distance and $2 \mathrm{~h}$ in LT), with the values color coded according to radius such that black corresponds to $3-6$, blue to $6-12$, green to $12-18$, orange to $18-24$, and red to 24 $30 \mathrm{R}_{\mathrm{S}}$. A two-term Fourier series fit of the form $\psi_{i}^{*}(\varphi)=$ $\psi_{i_{0}}^{*}+\psi_{i_{1}}^{*} \cos \left(\varphi-\varphi_{i}\right)$ is then computed for these data, where the three constants $\psi_{i_{0}}^{*}, \psi_{i_{1}}^{*}$ and $\varphi_{i}$ have been deter- mined using a simple linear-least squares algorithm. These fits are shown in the top plots of Figure $7 \mathrm{~b}$ by the similarly color coded solid lines, yielding the normalized angular velocity profiles shown in the bottom plots of Figure $7 \mathrm{~b}$ when substituted into equation (12b).

[39] Examining first the results for the $\theta$ field component shown in the middle plots of the figure, it can be seen that the results in the radial ranges 6-12 (blue) and 18-24 $\mathrm{R}_{\mathrm{S}}$ (orange) are in conformity with expectations based on Figure 5, namely smaller angular velocities falling to minima of $\sim 0.7 \Omega_{M}$ in the dusk and postdusk sectors, and larger angular velocities peaking at $\sim 1.5-2 \Omega_{M}$ in the dawn sector. Differing variable results occur in the radial ranges 3-6, 12 18 , and $24-30 \mathrm{R}_{\mathrm{S}}$ (black, green and red traces) where the rather scattered phase values are not well-modeled by the two-term Fourier fit.

[40] Similar results to the blue and orange curves in the $\theta$ component are observed in the left plots of Figure $7 \mathrm{~b}$ for the $r$ field component, except that the minima and maxima are typically shifted to later LT by $\sim 3 \mathrm{~h}$ compared with the $\theta$ component. In the innermost region (black trace) the minimum angular velocity occurs at $\sim 15 \mathrm{~h} \mathrm{LT}$ and the maximum at $\sim 03 \mathrm{~h} \mathrm{LT}$, while in the outer regions the minima occur at $\sim 21 \mathrm{~h} \mathrm{LT}$ and maxima at $\sim 09 \mathrm{~h} \mathrm{LT}$ (except for the $18-24 \mathrm{R}_{\mathrm{S}}$ range shown by the orange trace where again the phase data are not well represented by the Fourier fit). We also note that the azimuthal gradient of $\psi_{i}^{*}$ becomes sufficiently large and negative at large radial distances $24-30 R_{S}$ (red traces) in the postdawn sector that the angular velocity given by equation $(12 \mathrm{~b})$ reverses sense to negative, indicating retrograde motion of the phase fronts in this sector. The negative values are too large to display in Figure 7b, but the interval where they occur is bounded by the red vertical lines in the angular velocity plot where $\Omega_{i} / \Omega_{M}^{*}$ passes through $\pm \infty$.

[41] Results for the $\varphi$ field component in the right plots of Figure $7 \mathrm{~b}$ show somewhat differing behavior, with low angular velocities throughout the nightside sector with consistently reducing minima with increasing radial distance. While the angular velocity in the innermost radial range 3-6 $\mathrm{R}_{\mathrm{S}}$ (black trace) remains close to $\Omega_{M}$, the slightly premidnight minima decrease from $\sim 0.7 \Omega_{M}$ for $6-12 R_{S}$ to $\sim 0.4 \Omega_{M}$ for $24-30 \mathrm{R}_{\mathrm{S}}$ (red trace). Phase speeds exceeding $\Omega_{M}$ are then found throughout the dayside, again increasing with radial distance, once more becoming retrograde in a band of LT centered near noon in the $18-30 \mathrm{R}_{\mathrm{S}}$ radial range (orange and red traces).

\section{Form of the Oscillating Field in the Equatorial Region}

[42] In order to visualize the overall structure of the oscillatory field implied by the amplitude and phase results presented in section 3, in Figure 8 we show plots of the oscillating field vectors in the equatorial plane at fixed values of $\Phi_{M}$ in $45^{\circ}$ increments, displaying the evolution of the field over a complete cycle. As in previous related figures, the Sun is to the right in each plot, with dusk at the top. The spatial bins used to construct the figure are identical to those in Figure 5 and Figure $6 c$, corresponding to $3 R_{S}$ bins in radial distance and $2 \mathrm{~h}$ in LT, computed every hour. The vectors shown in Figure 8 are derived from the $r$ and $\varphi$ field 

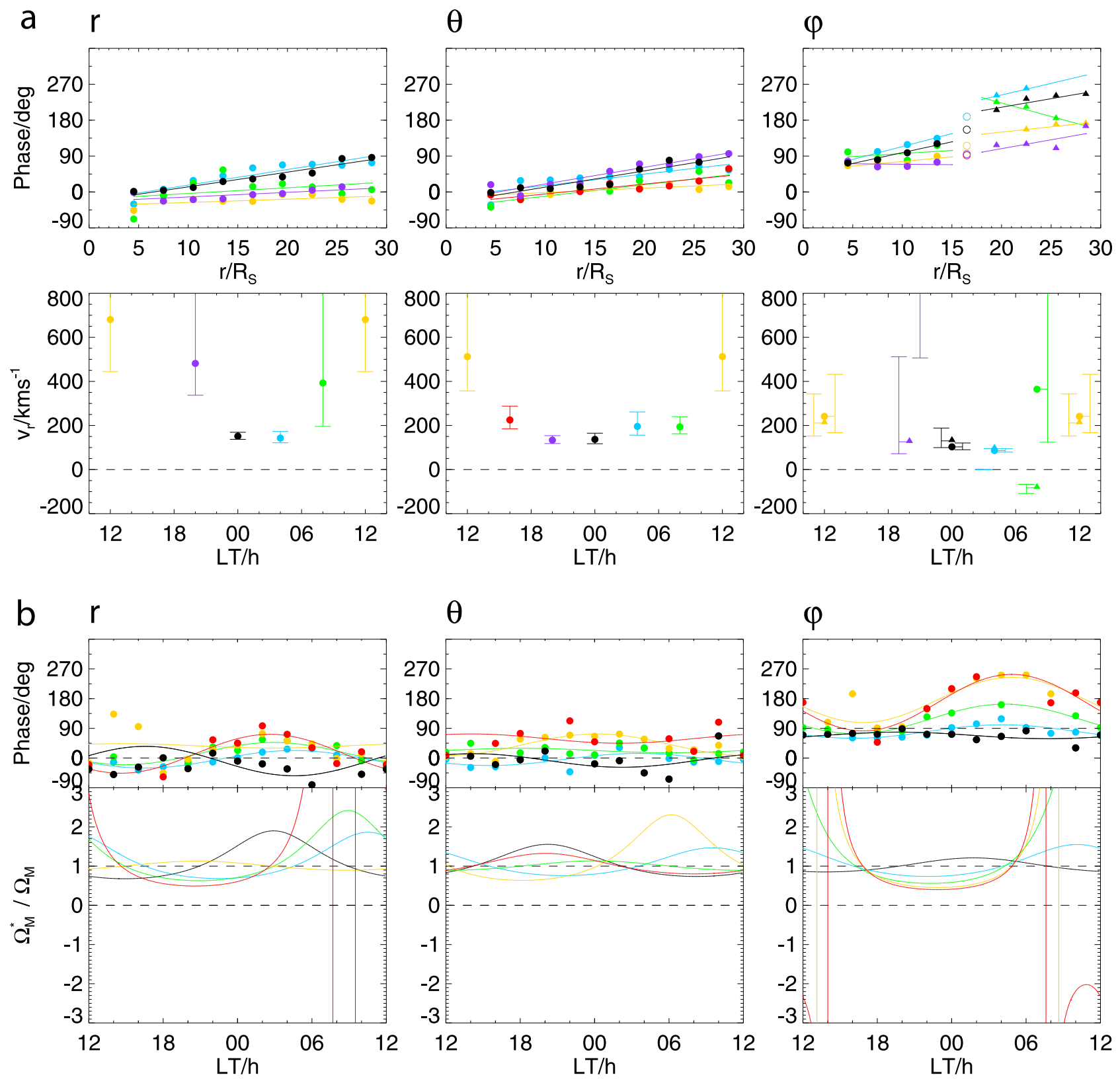

Figure 7. (a) Plots related to the radial phase velocity discussed in section 4 . The top plots show the phase $\psi_{i}^{*}$ plotted versus radial distance for each field component ( $r, \theta, \varphi$ from left to right), in a format similar to the bottom plots of Figure $4 \mathrm{a}$, where the color coding indicates the LT range as in the latter figure. Here, the similarly color coded solid lines show linear fits to these data (piecewise for the $\varphi$ field component data as described in section 4, omitting data in the 15-18 $\mathrm{R}_{\mathrm{S}}$ radial range shown by the open circles). Radial phase velocities determined from the gradients of these fits using equation (12a) are then plotted versus LT in the bottom plots, where the error bars are determined from the $1-\sigma$ uncertainties in the gradient of the fit. The velocities determined in the inner and outer region in the $\varphi$ field component are indicated by solid circles and triangles, respectively, with the error bars offset to the right and left. (b) Plots related to the azimuthal phase velocity discussed in section 4 . The top plots show the phase $\psi_{i}^{*}$ plotted versus LT for each field component in a format similar to the bottom plots of Figure $4 \mathrm{~b}$, where the color coding indicates the radial distance as in the latter figure. The similarly color coded solid lines show a two-term Fourier series fit to these data. The bottom plots show the corresponding azimuthal phase velocities versus LT in each radial range, expressed as a fraction of the angular frequency of the core phase model as given by equation (12b), color coded in the same format as the top plots. Regions in which the azimuthal gradient in the two-term Fourier series fit becomes sufficiently large and negative that the normalized angular velocity becomes negative, indicating retrograde motion, are bounded by the vertical lines in the bottom plots where the function passes through $\pm \infty$. 
a) $\Phi_{\mathrm{M}}=0^{\circ}$
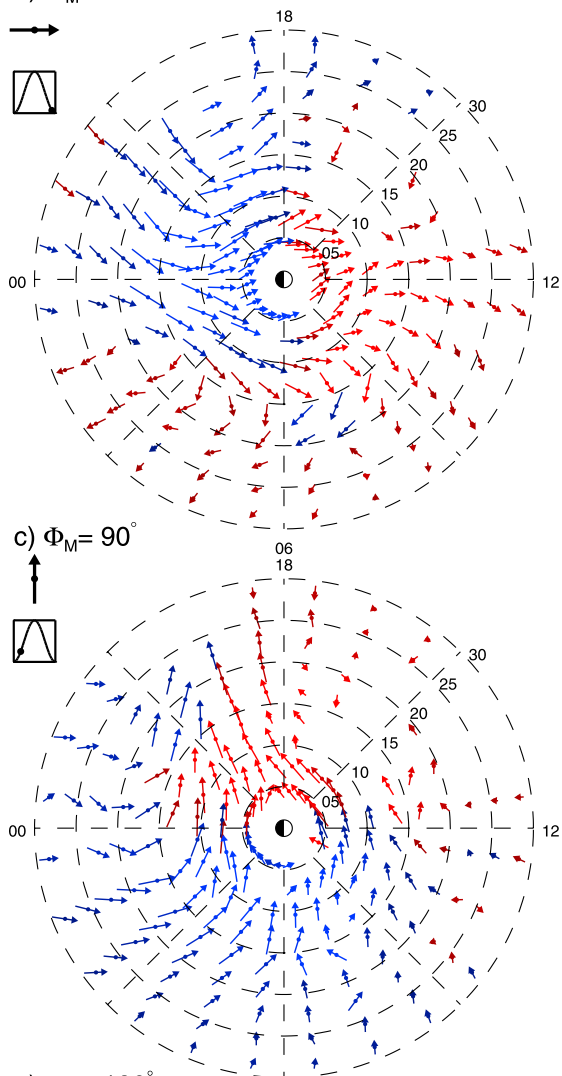

e) $\Phi_{M}=180^{\circ}$

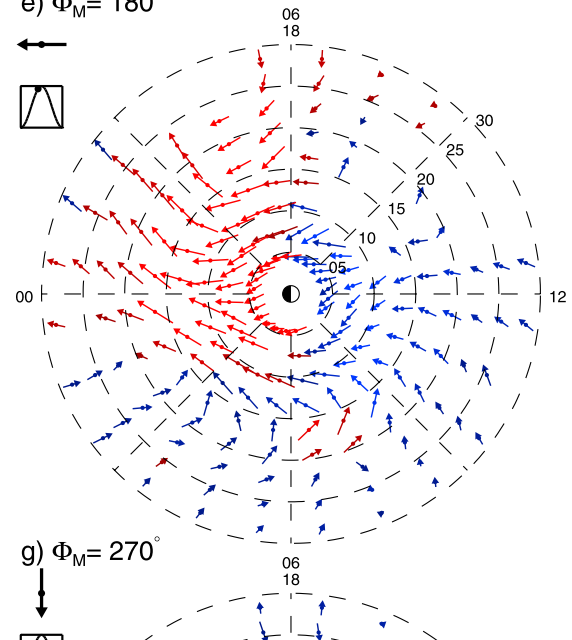

V

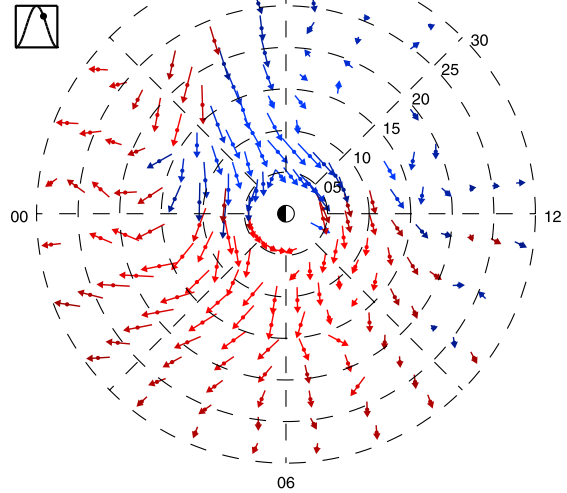

b) $\Phi_{\mathrm{M}}=45^{\circ}$

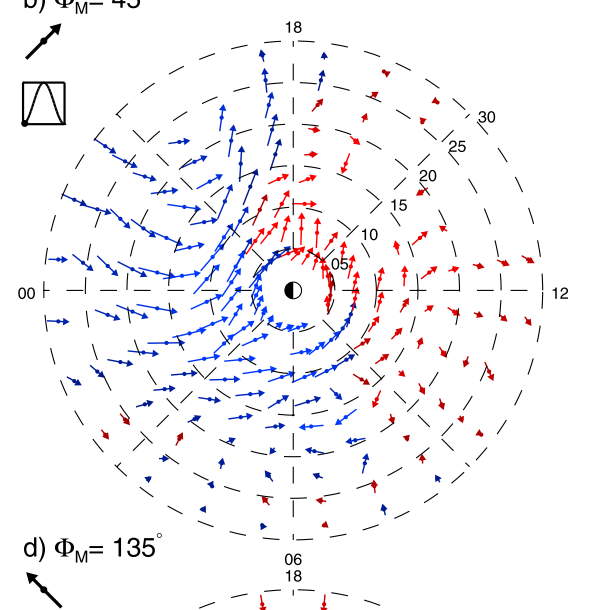

$\Delta$
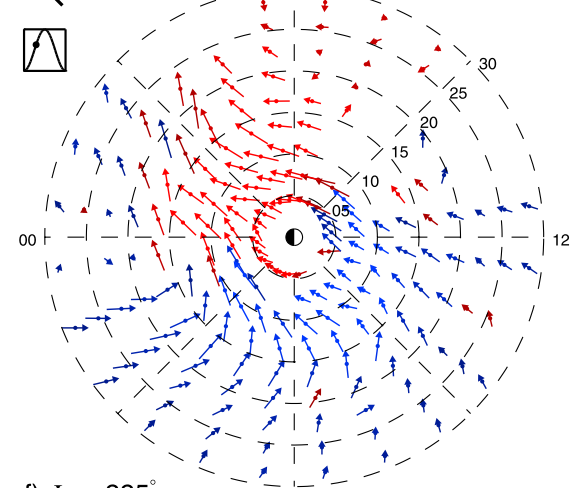

f) $\Phi_{M}=225^{\circ}$

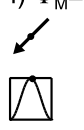

$-1-$
06
18
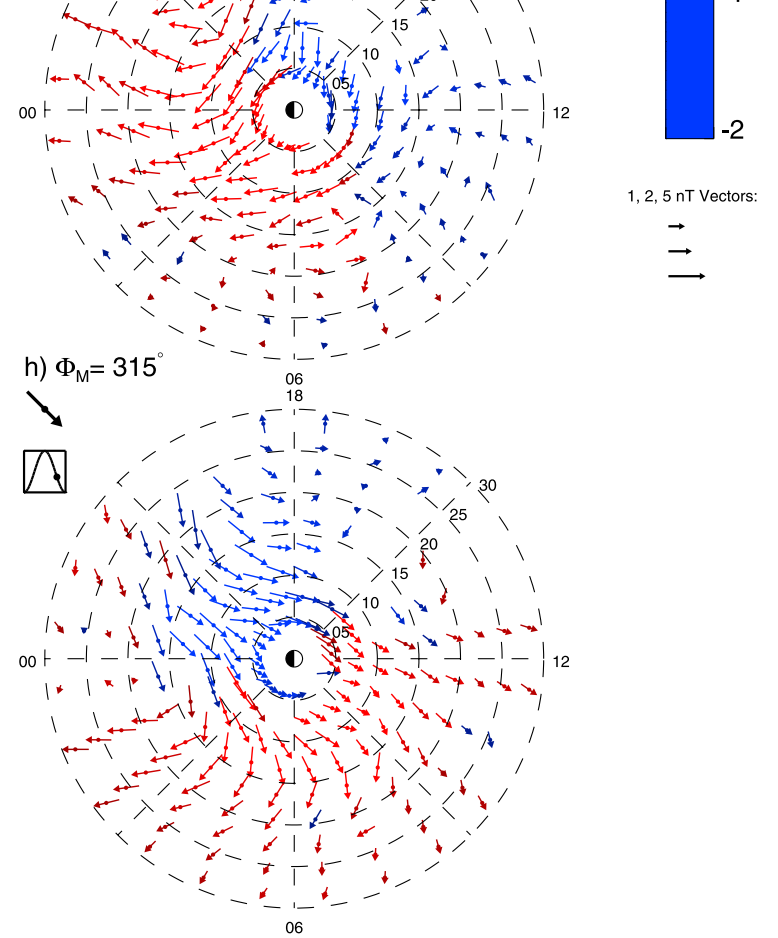

Figure 8

15 of 28 
component data, plotted at the center of each bin using a nonlinear length scale as indicated on the right-hand side of the figure, such that the orientation of the field vectors is still discernible when the field amplitude is small. The vectors themselves are color coded, indicating the sign and magnitude of the $\theta$ field component oscillations, with reds corresponding to positive values (pointing southward into the plane of the figure), and blues to negative values (northward out of the plane of the figure), according to the color scale shown on the right of the figure. The oscillation phase, $\Phi_{M}$, is indicated in the top left corner of each plot, together with an arrow showing the expected orientation of the core field according to the Provan et al. [2009a] model (equation (1)). The second icon below the core field arrow in each plot indicates the approximate SKR power modulation phase, given by $\Phi_{S K R} \approx \Phi_{M}+150^{\circ}$ according to equation (2b) with $\psi_{M}=150^{\circ}$ as discussed in section 2 , with SKR maxima occurring when $\Phi_{S K R}=360 \mathrm{~N} \mathrm{deg}$. The trace plotted in this icon is a simple cosine, with the position of the overplotted solid circle indicating the instantaneous phase of each plot relative to SKR maxima and minima, with SKR maximum occurring when the solid circle reaches the peak of the cosine. The SKR maximum at $\Phi_{M} \approx 210^{\circ}$ occurs close to the conditions shown in Figure $8 \mathrm{f}$ for $\Phi_{M}=225^{\circ}$, while SKR minimum correspondingly occurs at $\Phi_{M} \approx 30^{\circ}$, close to the conditions shown in Figure $8 \mathrm{~b}$ for $\Phi_{M}=45^{\circ}$. We note, of course, that by virtue of the cosine fit to the data given by equation (10), Figures $8 \mathrm{a}-8 \mathrm{~d}$ are related to the Figures $8 \mathrm{e}-8 \mathrm{~h}$ simply by a reversal in the direction of each individual field vector (and an inversion of their color), but it is useful to include these plots such that the oscillation can be viewed over a complete cycle. Additionally, we animate the data presented in Figure 8 at $2^{\circ}$ increments of $\Phi_{M}$ in Animation S4 as part of the auxiliary online material.

[43] We first examine Figure 8a, for which $\Phi_{M}=0^{\circ}$ such that the core field is expected to point toward the Sun. It can be seen that this is generally the case out to $\sim 15 \mathrm{R}_{\mathrm{S}}$, but with a pronounced "deflection" of the field vectors around the planet now evident in this format, together with an overall exclusion of the equatorial field from the inner region. In terms of the previous discussion, these effects are a consequence of the increasing dominance of the $\varphi$ field component relative to $r$ inside of $\sim 9 \mathrm{R}_{\mathrm{S}}$ noted in section 3, combined with an overall weakening of both components in the inner region. In the outer region, the dominant nightside $r$ field component points inward in a narrow range of LT in the premidnight sector (the green and blue regions in the top plot of Figure 5a), and outward at other LTs, while the approximately antiphase $\varphi$ component is positive (directed clockwise) in a narrow LT sector near midnight (the red and orange regions in the top plot of Figure 5c) and is negative elsewhere. Overall the field in the equatorial plane points inward in the premidnight sector, around the planet via dusk and dawn to the dayside and then "returns" via major circulations of the field vectors in the outer regions at dusk and dawn. The circulation cell at dusk is associated with an anticlockwise "semicircular" rotation of the field vectors beyond $\sim 15 \mathrm{R}_{\mathrm{S}}$ that is centered near $\sim 20 \mathrm{~h} \mathrm{LT}$ (within the limitations imposed by the data coverage available in this sector), while the clockwise field circulation at dawn takes the form of a more "sheet-like" shear in the field vectors across $\sim 15 \mathrm{R}_{\mathrm{S}}$ that extends from $\sim 3$ to $9 \mathrm{~h} \mathrm{LT}$, associated with the rapid phase increase in the $\varphi$ field component in this sector, as noted in section 3.1. Consideration of Ampère's law indicates that the anticlockwise field circulation in the dusk sector is associated with a northward current flow through the equatorial plane, while the clockwise circulation near dawn is associated with a southward current flow. We also note that the field exclusion effect in the inner region also implies NorthSouth current flows at dusk and dawn, directed opposite to that in the adjacent outer region. Both inner and outer North-South currents will be quantified in section 6 below. With regard to the oscillation in the $\theta$ field component, we note that this is largely negative (northward) in the dusk and midnight sector (blue and green regions in top plot of Figure $5 \mathrm{~b}$ ), and positive elsewhere.

[44] We now consider how this pattern evolves over a complete oscillation cycle. In Figure $8 \mathrm{~b}$ for $\Phi_{M}=45^{\circ}$ the field in the interior region has rotated to point duskward of the Sun as expected, the anticlockwise cell near dusk has rotated somewhat toward midnight, and the clockwise shear region near dawn has extended into the prenoon sector. Further evolution to $\Phi_{M}=90^{\circ}$ as depicted in Figure 8c results in largely duskward directed fields becoming present throughout the equatorial region investigated, with the outer anticlockwise circulation cell becoming broadened and centered in the midnight sector, while the clockwise circulation on the dayside has weakened substantially and all but disappeared. In Figure $8 \mathrm{~d}$ for $\Phi_{M}=135^{\circ}$ the anticlockwise circulation cell now intrudes into the dawn sector, beginning to form a shear layer of the opposite rotation sense than was present at the start of the cycle, while indications of clockwise circulation appear near dusk. These features develop further in Figure 8e for $\Phi_{M}=180^{\circ}$, where the vectors are directed exactly opposite to those in Figure 8a

Figure 8. Plots showing the evolution of the oscillatory magnetic field in the equatorial plane over a complete cycle, as discussed in section 5. The magnetic phase $\Phi_{M}$ is $0^{\circ}$ in Figure $8 \mathrm{a}$, and increases by $45^{\circ}$ in each subsequent panel, up to $315^{\circ}$ in Figure 8h. The main plot in Figures $8 \mathrm{a}-8 \mathrm{~h}$ show the equatorial field vectors derived from the $r$ and $\varphi$ component amplitude and phase data, where the vectors are shown using a nonlinear scale indicated on the right of the figure. The color of each vector indicates the sense and magnitude of the $\theta$ component according to the color scale on the right of the figure, with reds indicating positive (southward directed), and blues negative (northward directed) field values. The icons in the top left corner of each plot indicate the expected orientation of the core equatorial field components according to the phase model of Provan et al. [2009a] (equation (5)), together with the approximate SKR phase according to equation (8a). The latter is indicated by a solid circle moving on a sinusoid such that the SKR power maximizes when the solid circle reaches the peak of the sinusoid. This condition occurs nearest to the field configuration shown in panel $(f)$, with SKR minimum correspondingly occurring nearest to panel $(b)$. Note that the field vectors in the lower four panels are exactly reversed in sense compared with the upper four panels, and with a reversed color code. 
as previously indicated. Subsequent evolution through Figures $8 \mathrm{f}-8 \mathrm{~h}$ is then identical to Figures $8 \mathrm{~b}-8 \mathrm{~d}$ but with field directions reversed.

[45] An important feature that can be discerned on careful inspection of Figure 8, but is more readily evident when viewed in Animation S4, concerns the polarization of the field oscillation in the equatorial plane. Over most of this region, the $\varphi$ field component is in approximate lagging quadrature with the $r$, resulting in rotation of the field vectors in the equatorial plane in the same sense as the planet, as noted by Espinosa et al. [2003a], Andrews et al. [2008], and Provan et al. [2009a] as discussed in section 1. More specifically, this sense of rotation applies when $0^{\circ}<\Delta \psi^{*}<$ $180^{\circ}$ in the right plot of Figure $6 \mathrm{c}$, where $\Delta \psi^{*}=\psi_{\varphi}^{*}-\psi_{r}^{*}$. However, in the outer dawn region beyond $\sim 15 \mathrm{R}_{\mathrm{S}}$ the phase difference instead satisfies $180^{\circ}<\Delta \psi^{*}<360^{\circ}$, under which condition the field vectors rotate in the opposite sense to planetary rotation. The region in which this condition is met in Figure 8 corresponds approximately to the orange region in the right plot of Figure 6c. We note that this sense of polarization corresponds to that of a rotating transverse dipole. Indeed, it can be seen from Figure 8 that the overall form of the equatorial field vectors in the outer region resembles that of a transverse dipole, the dipole moment concerned being that of the outer current system flowing North-South through the equatorial plane, as will be discussed further in section 6 .

\section{North-South Currents in the Equatorial Plane}

[46] As just discussed in relation to Figure 8, the circulation of the $r-\varphi$ oscillation field vectors in the equatorial plane implies the presence of currents flowing North-South through the plane, as does the deflection and weakening of the core field in the inner region. Applying Ampère's law in spherical polar coordinates to the equatorial plane $\left(\theta=90^{\circ}\right)$ yields the following expression for the colatitudinal component of the equatorial current density

$$
j_{\theta}(r, \varphi, t)=\frac{1}{\mu_{0} r}\left(\frac{\partial B_{r}}{\partial \varphi}-\frac{\partial}{\partial r}\left(r B_{\varphi}\right)\right),
$$

which we apply here to the oscillatory field only, thus yielding values of the oscillatory current density. We note that at least within the equatorial quasi-dipolar core region within $\sim 15 \mathrm{R}_{\mathrm{S}}$, the nonoscillatory background field is also principally directed North-South, so that the current density given by equation (13) is essentially the field-aligned component. The outer portion of these currents are thus expected to relate to the field-aligned current systems discussed by Southwood and Kivelson [2007] and Provan et al. [2009a] and are thus of direct significance to the origin of these oscillatory phenomena. Outside these distances, however, in the magnetodisc region where radial fields are also important, the North-South currents may also generally contain some field-perpendicular component. While it would also be of interest to examine the other current components flowing in the equatorial plane, these currents depend not only the variations of the $\theta$ field component with radial distance and LT, but also on the undetermined variations of the $r$ and $\varphi$ field components with colatitude. Therefore these currents cannot be calculated from the field oscillation data determined in this paper.

[47] The North-South current density has been calculated from equation (13) using a centered finite difference scheme in which the amplitude and phase of the oscillatory $r$ and $\varphi$ field components have first been calculated in a set of overlapping bins that are each of width $3 \mathrm{R}_{\mathrm{S}}$ in radial distance and $2 \mathrm{~h}$ in LT, as above, computed every hour in LT. For the $\varphi$ field component the radial bins are 3-6, 6-9, .., 27-30 $\mathrm{R}_{\mathrm{S}}$, identical to those employed in creating Figure 8, such that field difference values for a given $\Phi_{M}$ corresponding to the $\partial\left(r B_{\varphi}\right) / \partial r$ term in equation (13) are evaluated at $6,9, \ldots$, $27 \mathrm{R}_{\mathrm{S}}$, at each $\mathrm{LT}$. The radial bins used to determine the oscillatory $r$ field component have then been displaced by $1.5 \mathrm{R}_{\mathrm{S}}$ to $4.5-7.5,7.5-10.5, \ldots, 28.5-31.5 \mathrm{R}_{\mathrm{S}}$, thus also being centered on $6,9, \ldots, 30 \mathrm{R}_{\mathrm{S}}$, with the values employed to determine $\partial B_{r} / \partial \varphi$ at that $\Phi_{M}$ being obtained from the bins displaced by $1 \mathrm{~h}$ of LT on either side of the bins used to determine the corresponding $\partial\left(r B_{\varphi}\right) / \partial r$ value. The difference values that are combined together in equation (13) to determine the current density are thus centered on the same point as each other in the equatorial plane, and employ adjacent, but nonoverlapping data sets to estimate the field gradients. We note that while the spatial resolution of this scheme is relatively modest, such that the structure of the current will not be fully resolved, nevertheless the implications of Ampère's law in integral form is that the total integrated current should be well determined, provided that the sense of the current does not vary strongly on spatial scales similar to the bins employed.

[48] Results are plotted in Figure 9 in a format similar to Figure 8, showing the evolution of the currents over a complete oscillation cycle. The current density determined in each bin is color coded according to the scale on the right, such that reds correspond to positive currents, directed southward into the plane of the figure, while blues correspond to negative currents directed northward out of the plane of the figure. Examination of the uncertainties in the current density values, dependent on the uncertainties in the amplitude and phase of the oscillating field discussed in section 2.3, indicates that typical uncertainties are $\sim \pm 2.5 \mathrm{pA} \mathrm{m}^{-2}$ (corresponding to $\sim 25 \%$ of $\sim 10.0 \mathrm{pA} \mathrm{m}^{-2}$ ). Accordingly, the color scale for the currents has been chosen such that uncertain values within $\sim 2.5 \mathrm{pA} \mathrm{m}^{-2}$ of zero are shown as near white. Bins in which the current density could not be calculated due to lack of data in one or more field components are colored gray. The value of the oscillation phase $\Phi_{M}$, the orientation of the core field according to the Provan et al. [2009a] model, and the approximate SKR modulation phase are indicated in the top left corner of each plot in a format identical to Figure 9. Examination shows that the currents derived here are predominantly associated with radial gradients in the azimuthal field (the second term in equation (13)). An exception to this occurs in the dusk and postdusk sectors, where the azimuthal gradients in the radial field are of similar magnitude.

[49] We first examine the currents shown in Figure 9a, corresponding to $\Phi_{M}=0^{\circ}$, together with the corresponding field vectors shown in Figure 8. Examination shows that, in broad terms, the currents can be divided into two radial regions, an innerregion corresponding to the two inner rings in the current density plots spanning 4.5-10.5 $\mathrm{R}_{\mathrm{S}}$, and an 
a) $\Phi_{M}=0$
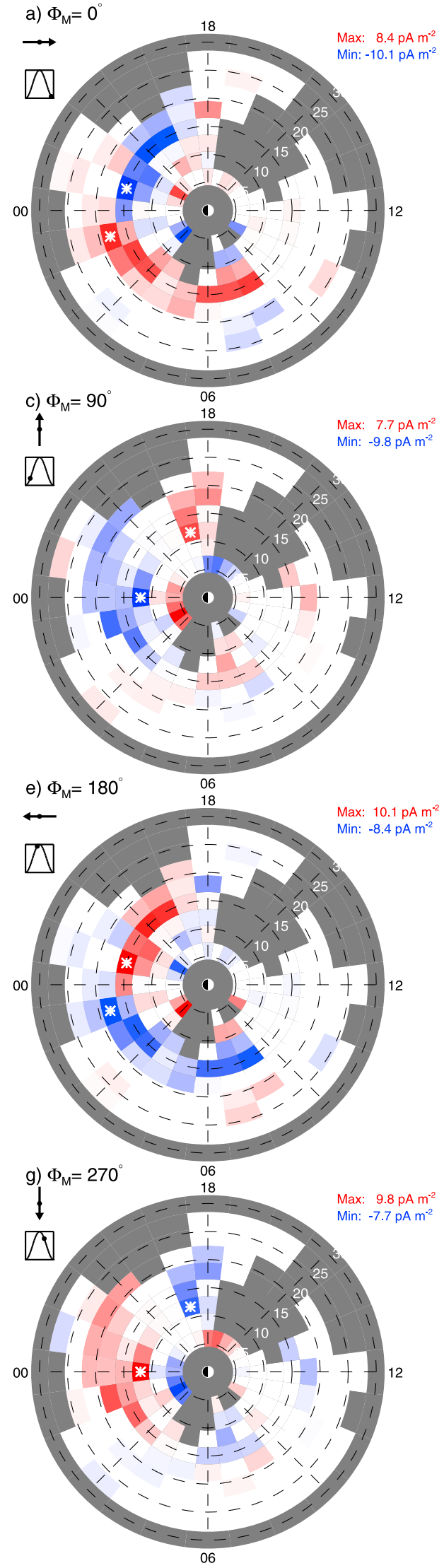
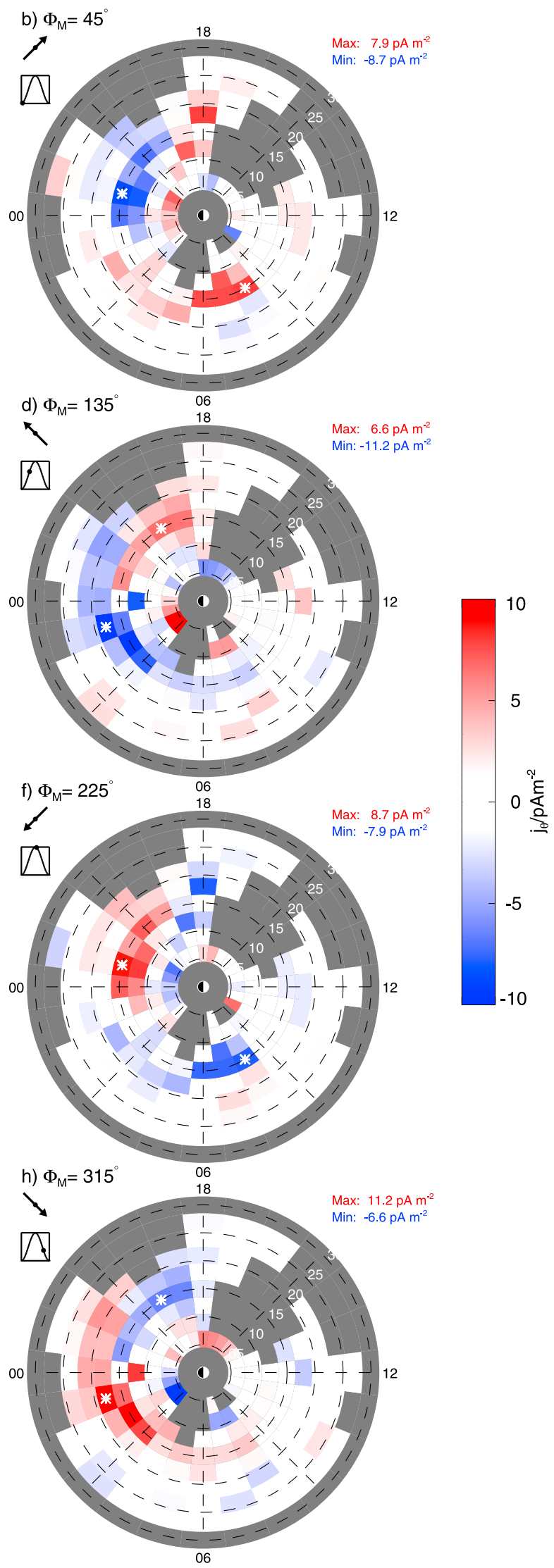

Figure 9 
outer region spanning $10.5-28.5 \mathrm{R}_{\mathrm{S}}$. The currents in the inner region relate to the deflection and weakening of the equatorial core field, being positive (southward) and negative (northward) at dusk and dawn, respectively, as anticipated. The currents in the outer region, however, are somewhat more complicated. The primary regions of positive and negative current take the form of broad spirals that extend clockwise from the inner region to the outer boundary. The region of negative current extends from the midnight sector to predusk, associated with the anticlockwise circulation in the field vectors that is evident in Figure 8 in this region, while the region of positive current similarly extends from midmorning to premidnight, associated with the strong clockwise shear in the field that is evident in the dawn sector. Bins containing the peak positive and negative current densities in the outer region are marked in Figure 9 with stars, with the values concerned being given in the top right hand corner of the plot. The peak current densities at $\Phi_{M}=0^{\circ}$ are $\sim 10 \mathrm{pA} \mathrm{m}^{-2}$ in each case, located at $\sim 15 \mathrm{R}_{\mathrm{S}}$ in the premidnight sector for the negative current, and in the postmidnight sector for the positive current. In addition to these primary current regions we also note the presence of secondary regions of weaker current that prove to be of significance in the overall evolution of the current system during the oscillation cycle. Specifically, a region of positive current is present near the dusk meridian (within the limitations of the data coverage in the afternoon sector), while a region of weak negative current is located postdawn at radial distances beyond $\sim 20 \mathrm{R}_{\mathrm{S}}$. Overall, therefore, we identify four current regions in the outer equatorial magnetosphere, two of each sign.

[50] We now consider Figures 9b-9e, showing how these current regions evolve over half an oscillation cycle. Alternatively, Animation S5 in the auxiliary online material provides an animation at $2^{\circ}$ intervals of $\Phi_{M}$. In the inner region it can be seen that the current pattern rotates approximately uniformly with the core field as expected, with the sense of the currents reversing on either side of the planet between $\Phi_{M}=0^{\circ}$ and $180^{\circ}$. In the outer region, however, it can be seen that the secondary region of positive current near dusk at $\Phi_{M}=0^{\circ}$ strengthens, rotates and expands to form the primary region of positive current at $\Phi_{M}=180^{\circ}$ extending from dusk to midnight, while the premidnight primary region of negative current at $\Phi_{M}=0^{\circ}$ similarly rotates and expands to form the primary region of negative current spanning midmorning to premidnight at $\Phi_{M}=180^{\circ}$. At the same time, the primary dawn-side region of positive current at $\Phi_{M}=0^{\circ}$ weakens and moves radially outward to form the secondary region of positive current at $\Phi_{M}=180^{\circ}$, while the dawn-side secondary region of negative current at $\Phi_{M}=0^{\circ}$ dissipates at large radial distances in the dawn sector and can no longer be discerned. The evolution of the current system in the second half-cycle shown in Figures 9e-9h then follows the same pattern, but with the sense of current reversed. Although the detailed behavior of the current in the outer region is somewhat obscured by lack of data coverage in the postnoon sector, it thus appears that we may regard each current region as first intensifying in the dusk sector over a broad radial range, then propagating in LT around the planet to form an extended clockwise spiral stretching from midmorning to premidnight via dawn after one cycle, and decaying at large radial distance in the dawn and dayside sectors during the following cycle. Each individual current region thus remains identifiable for two complete cycles, with an angular frequency of rotation which is roughly half that of the core field. The properties of the current system associated with the field oscillations thus appears to be somewhat more complex than envisaged by Southwood and Kivelson [2007] and Provan et al. [2009a].

[51] In simple terms, the above behavior of the NorthSouth currents results from the fact that in the outer region the azimuthal variation of the field has a significant component with azimuthal wave number $m=2$, giving rise to two current regions of each sign, one major and one minor, in addition to the $m=1$ variation that is dominant in the core. We note that our model only specifies the period of the oscillation, $2 \pi / \Omega_{M}$, and not the spatial scale of its structure (except that the data are averaged in bins of finite size), the latter being wholly determined from independent fits to the spatially binned data. Thus while the primary variations with $m=1$ are described by the function $\exp \left[i\left(\Omega_{M} t-\varphi\right)\right]$, with consequent azimuthal phase speed $\Omega_{M}$, the secondary variations with $m=2$ are described by the function $\exp \left[i\left(\Omega_{M} t-\right.\right.$ $2 \varphi)]$ (which still varies at the model period $2 \pi / \Omega_{M}$ ), with an azimuthal phase speed of $\Omega_{M} / 2$.

[52] In Figure 10 we further quantify the evolution of the current regions discussed qualitatively above. At given values of the oscillation phase $\Phi_{M}$ we have divided the equatorial plane into six contiguous current regions, two in the inner region and four in the outer, in accordance with the above discussion. These are shown on the left side of Figure 10 at $10^{\circ}$ intervals of $\Phi_{M}$ between $0^{\circ}$ and $170^{\circ}$ in a format similar to Figure 9 In the inner region the positive and negative current regions are shown as red and blue, respectively, while in the outer region the two positive current regions are shown as red and orange, and the two negative current regions as blue and green. The interface between the inner and outer regions is taken to lie at $10.5 \mathrm{R}_{\mathrm{S}}$ at the interface of the second and third radial bins, as indicated by the white dashed circle in the figure. To follow the evolution beyond $\Phi_{M}=170^{\circ}$ we return to $\Phi_{M}=0^{\circ}$ and switch colors, e.g., from red to blue and vice versa in the inner region. In the outer region the red current region at $170^{\circ}$ transforms to blue at $0^{\circ}$, blue transforms to orange, orange to green, and green

Figure 9. Plots showing the North-South current density in the equatorial plane computed from equation (13) using a finite difference scheme as outlined in section 6, where the Figures $9 \mathrm{a}-9 \mathrm{~h}$ correspond to the same phases as those in Figure 8 , namely $\Phi_{M}=0^{\circ}$ to $315^{\circ}$ in $45^{\circ}$ steps. The color scale, as given on the right of the figure, indicates the current density determined in each bin, with reds corresponding to positive currents (southward directed) and blues to negative (northward) currents. Grey regions indicate bins for which the current density cannot be computed due to the lack of field component data. The bins in which the current densities in the outer region (beyond radial distances of $10.5 \mathrm{R}_{\mathrm{S}}$ ) are maximally positive and negative are indicated by white stars, with the corresponding peak positive and negative current densities being given in the top right corner of each panel. The icons in the top left corner of each plot are the same as in Figure 8. 

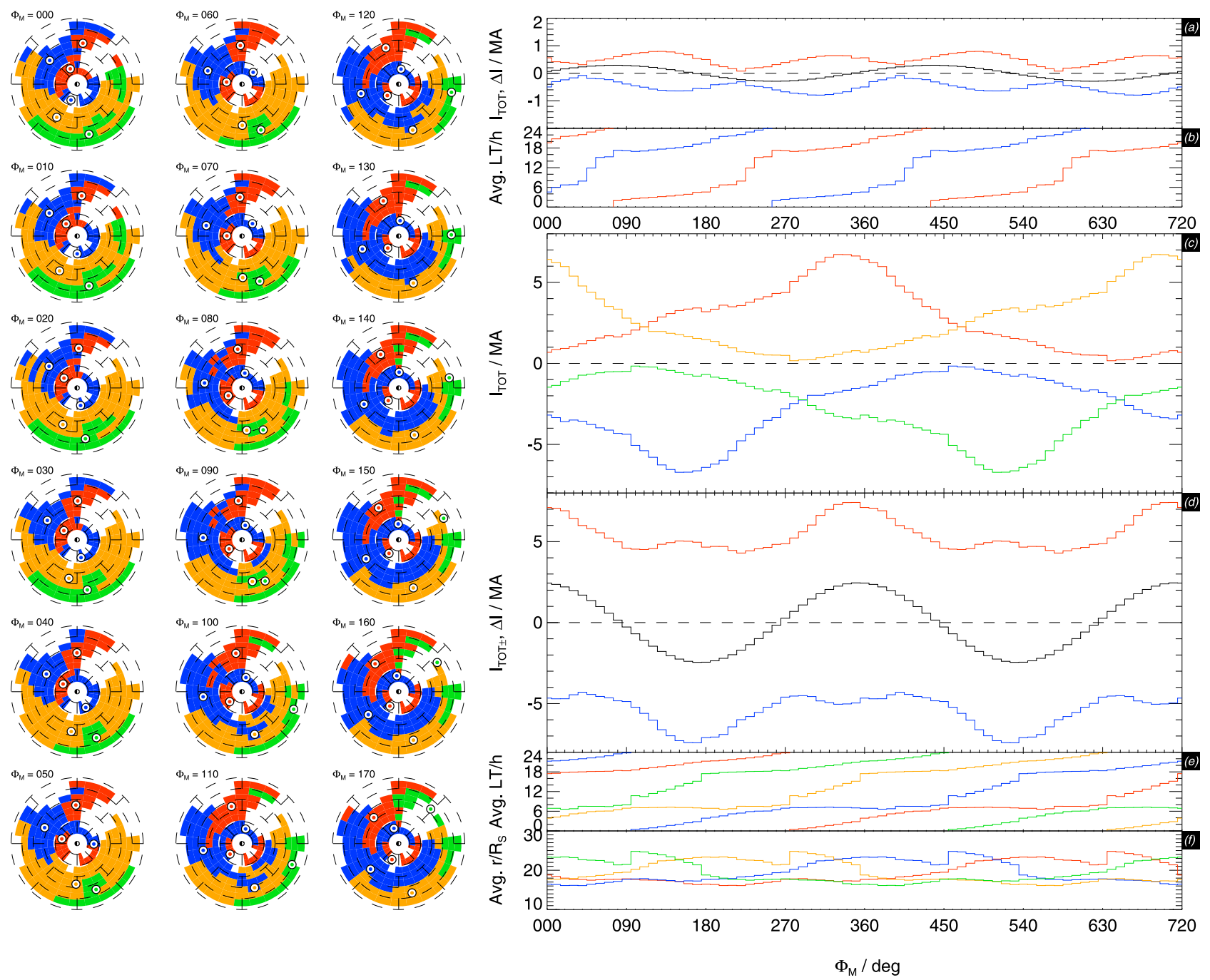

Figure 10. The diagrams on the left of the figure color code the six regions of North-South current flowing through the equatorial plane at $10^{\circ}$ increments of $\Phi_{M}$ between $0^{\circ}$ and $170^{\circ}$ as discussed in section 6 , two in the inner region, four in the outer region, the dividing line between them at $10.5 \mathrm{R}_{\mathrm{S}}$ being shown by the white dashed circle. The format of these plots is similar to Figures 8 and 9, such that noon is to the right and dusk at the top. In the inner region, the red area indicates positive (southward) current, while the blue indicates negative (northward) current. In the outer region, the red and orange areas indicate positive current, while the blue and green indicate negative current. The evolution of each area between $\Phi_{M}=180^{\circ}$ and $350^{\circ}$ can then be obtained by suitable anticlockwise transformations of the colors shown. The current-weighted average radial distance and LT of each region are shown as color coded dots in the left plots and are plotted versus $\Phi_{M}$ over two cycles in the right panels together with the summed currents flowing through these regions. From top to bottom the latter panels show (a) the summed positive (red) and negative (blue) currents flowing in the inner region, together with their difference (black), (b) the averaged LT of the inner current regions, (c) the summed currents in the four outer regions using the same color code as in the left plots (for $\Phi_{M}=0^{\circ}$ to $170^{\circ}$ ), (d) the total positive (red) and negative (blue) current flowing in the outer region, together with their difference (black), (e) the averaged LT of the four outer current regions, and (f) the averaged radial distance of the outer current regions.

to red. Thus each outer current region can be followed using Figure 10 over the two full cycles of its evolution. We note that while the identification of the current regions is reasonably clear when the current is strong, it becomes more subjective where the currents are weaker and more spatially structured (e.g., the outer dawn sector). However, the latter weaker current regions are of lesser significance in the overall discussion.
[53] For each individual current region at each $\Phi_{M}$ we have computed the position of its center (as a currentweighted mean radial distance and LT), and have also summed the total current through its area. These quantities are shown on the right side of Figure 10, plotted versus $\Phi_{M}$ over two full oscillation cycles (since each individual outer current region evolves over two full cycles), while the center position data are also shown as colored dots in the equatorial 
plane plots on the right side of the figure. Results for the two inner current regions (whose full evolution occurs over one cycle) are shown in Figures 10a and 10b, specifically for the total current and averaged LT. The mean radial distance of the current regions are not shown in this case, since these are essentially invariant at $\sim 7.5 \mathrm{R}_{\mathrm{S}}$. The red lines in these panels refer to the region of positive (southward) current, while the blue lines refer to the region of negative (northward) current. First examining panel Figure 10a, it can be seen that the total positive and negative currents typically lie between $\sim 0.5 \mathrm{MA}$ and $\sim 1 \mathrm{MA}$, with two maxima per cycle near $\Phi_{M} \sim 125^{\circ}$ and $315^{\circ}$, and two minima near $\sim 45^{\circ}$ and $225^{\circ}$. Examination shows that the oscillatory nature of the currents is due to a combination of physical and accidental features of our data, relating to the spatial variation in the oscillatory fields, particularly the LT dependence in the $\varphi$ component shown in the right panels in Figure $4 \mathrm{~b}$, and the position of data gaps. The difference between the two currents is shown by the black line in Figure 10a, which oscillates as a strict sinusoid (as must be the case for the net current through a fixed area in the presence of sinusoidally varying fields) with an amplitude of $\sim 0.5$ MA. This net current must be due to overall current closure taking place either inside or outside the radial range considered, or within the regions containing data gaps. The averaged LT of the currents shown in Figure 10b generally increase approximately uniformly with phase $\Phi_{M}$, completing one rotation in each oscillation cycle, but show a pronounced "jump" at $\sim 240^{\circ}$ for the positive current region and at $\sim 60^{\circ}$ for the negative current region, which occur as these regions cross the data gap in the afternoon sector.

[54] Results for the outer current regions are shown Figures 10c-10f. Figure 10c shows the integrated currents in the four outer regions color coded as in the left-hand diagrams, Figure 10d shows the total summed positive (red) and negative (blue) currents and their difference (black), while Figures $10 \mathrm{e}$ and $10 \mathrm{f}$ show the averaged radial distance and LT of each region, respectively. We first focus on the evolution of one specific current region, that shown by the red line in Figures 10c, 10e, and 10f, corresponding to the red region in the left-hand plots during the $\Phi_{M}$ interval from $0^{\circ}$ to $170^{\circ}$ (followed by the blue region for $\Phi_{M}$ from $180^{\circ}$ to $350^{\circ}$ and so on). At $\Phi_{M}=0^{\circ}$ this region is centered near dusk at $\sim 18 \mathrm{R}_{\mathrm{S}}$, and carries a total current of $\sim 0.5 \mathrm{MA}$. The current in this region then rises near-monotonically to $\sim 6.5 \mathrm{MA}$ over the following cycle, while rotating to $\sim 3 \mathrm{~h} \mathrm{LT}$ via midnight at an approximately constant averaged radial distance. Over the next cycle the current then nearmonotonically declines to its initial value, while the center of the region increases its radial distance to $\sim 24 R_{S}$ in the dawn sector. We note that the orange, blue, and green lines in these panels all follow exactly the same behavior, with appropriate changes in the phase and in the sign of the current. Considered together, it can be seen that the outer current system can be approximately described in terms of two main current regions of opposite sign in some ranges of $\Phi_{M}$, such as the orange and blue current regions at $\Phi_{M}=0^{\circ}$, and the red and blue current regions at $\Phi_{M}=180^{\circ}$. At other phases, such as near $\sim 90^{\circ}$ and $\sim 270^{\circ}$ the current region can more appropriately be described in terms of three main current regions, a region of one sign centered near local midnight, sandwiched between two regions of opposite sign centered near dawn and dusk. We note that the former condition applies when the core equatorial field is directed approximately toward or away from the Sun, while the latter condition applies when the core field points approximately to dawn or dusk. Turning finally to the summed positive and negative currents in the outer region shown in Figure 10d, it can be seen that these vary more modestly in magnitude between minimum values of $\sim 4.5 \mathrm{MA}$ and maximum values of $\sim 7.5 \mathrm{MA}$, with a sinusoidally-varying difference of $\sim 2 \mathrm{MA}$ amplitude. We note that these currents (and their difference) are thus considerably larger than the currents in the inner region.

\section{Summary of Results}

[55] In this section we summarize and comment further on the main results reported above. In common with the earlier discussions, e.g., of Cowley et al. [2006] and Southwood and Kivelson [2007] it has been found that the properties of the equatorial oscillatory fields can be divided into inner and outer radial regions at a radial distance of $\sim 15 R_{S}$. This division also approximately separates the inner region dominated by the near-dipolar planetary field, referred to here as the core region of the magnetosphere, from the outer region dominated by the fields of the magnetodisc and nightside plasma sheet [Bunce et al., 2008; Arridge et al., 2008]. A major distinctive feature of the inner region is the presence of few-nT oscillations in the radial and azimuthal components of the field, that have commonly been described as forming an approximately uniform magnetic field in the equatorial plane that rotates in the sense of planetary rotation near the planetary period [e.g., Espinosa et al., 2003a]. The implication of such a description is that the oscillation amplitudes of the two field components are spatially uniform in this region, equal to each other, and with the phase of the azimuthal component in lagging quadrature with the radial component. Our results show that while the quadrature phase relationship is maintained to a good approximation throughout the inner region, the amplitudes of the components generally differ significantly from each other, and varying strongly with both radial distance and LT. Specifically, the amplitudes generally grow with radial distance from small values in the innermost region (3-6 $\mathrm{R}_{\mathrm{S}}$ ), are larger on the nightside than on the dayside, and with the azimuthal component exceeding the radial out to $\sim 12 \mathrm{R}_{\mathrm{S}}$ and vice versa beyond. Rather than forming a quasi-uniform field in the inner region, therefore, the equatorial field vectors inside $\sim 12 \mathrm{R}_{\mathrm{S}}$ are instead deflected around the innermost region, and are strongly suppressed in magnitude inside $\sim 6 \mathrm{R}_{\mathrm{S}}$, and on the dayside, compared with the nightside. These results thus suggest the presence of a rotating equatorial field that is principally generated outside $\sim 6 \mathrm{R}_{\mathrm{S}}$, with largest amplitudes on the nightside, and is suppressed inside that distance. The field suppression and deflection effects imply the presence of a rotating system of field-aligned currents flowing North-South through the equatorial plane which reduces the rotating field within their interior, the currents being directed northward on one side of the planet where the azimuthal field is positive, and southward on the other where the azimuthal field is negative. We have estimated the total field-aligned current in these regions to be $\sim 1 \mathrm{MA}$, flowing principally in the radial range inside $\sim 7.5 \mathrm{R}_{\mathrm{S}}$, thus corresponding to the Enceladus plasma torus 


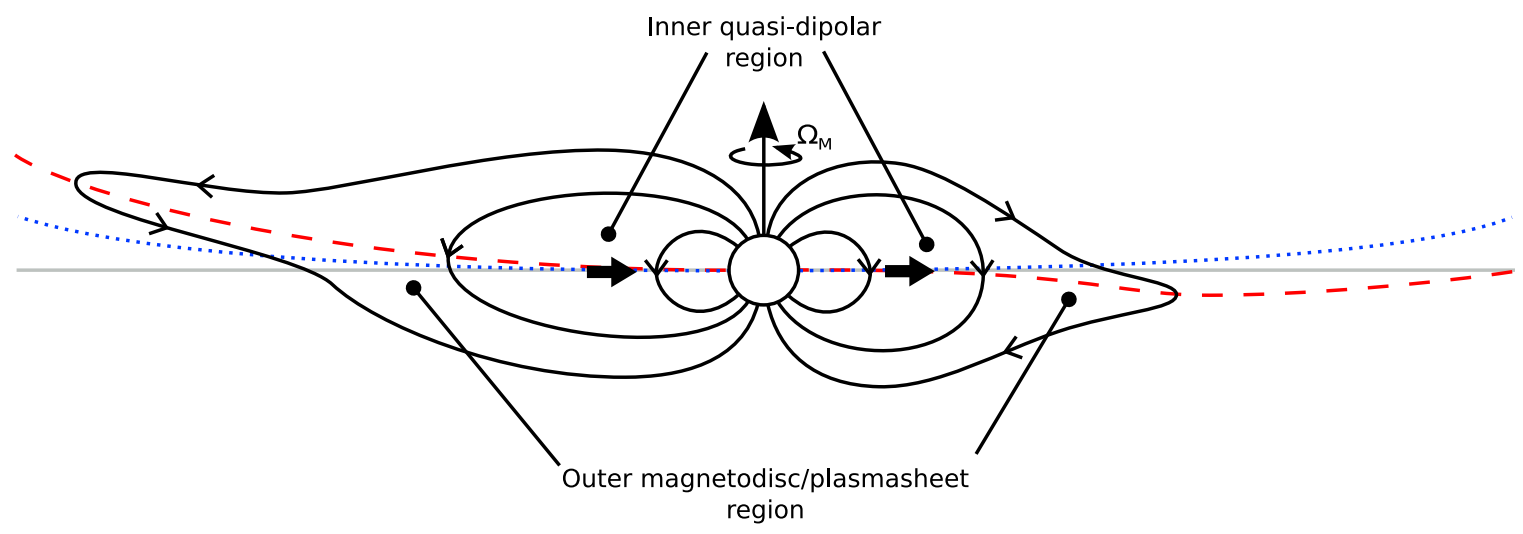

Figure 11. Meridional sketch of field lines in Saturn's magnetosphere, showing the displacement of the magnetic equator out of the planet's equatorial plane, for the situation in which the oscillatory radial field points from left to right (solid black arrows). The planet's spin equatorial plane is shown by the grey solid line, while the averaged position of the magnetic equator is shown by the blue dotted line, being everywhere above the equatorial plane for the southern summer conditions prevailing during the interval of this study Arridge et al. [2008]. The instantaneous magnetic equator is shown by the red dashed line. At the instant depicted, the magnetic equator is displaced northward of its mean position where the radial field points inward, and southward of its mean position where the radial field points outward. The pattern then rotates in the same sense as the planet at the magnetospheric period $2 \pi / \Omega_{M}$, such that a stationary observer will observe a periodic North-South "flapping" motion of the magnetic equator.

lying inside the main hot plasma ring current region [e.g., Young et al., 2005; Bunce et al., 2007; Schippers et al., 2008]. We note that these field lines map to Saturn's ionosphere at latitudes of $\sim 68^{\circ}$, thus typically lying well equatorward of Saturn's auroral oval centered near $\sim 75^{\circ}$ [e.g., Badman et al., 2006].

[56] Outside this innermost current system, the oscillatory radial and azimuthal fields, directed essentially transverse to the North-South quasi-dipolar planetary field, result in oscillatory tilting of the magnetospheric field lines. As a consequence, the magnetic equator becomes displaced south of the planetary dipole equator where the oscillatory radial field is positive, and north of the dipole equator where the radial field is negative. This is illustrated in Figure 11, where we show a sketch of the field lines in a meridian plane at an instant when the oscillatory equatorial field points from left to right as indicated by the horizontal solid arrows. The magnetic equator, where the sign of the total radial field reverses, is shown by the dashed line. In effect, beyond $\sim 6 \mathrm{R}_{\mathrm{S}}$ the northward normal to the magnetic equator is tilted toward the direction of the equatorial field (to the right in Figure 11), approximately toward azimuth $\Phi_{M}(t)$ according to equation (5), and rotates with the oscillatory field at the period given by equation (3). Further discussion of Figure 11 with regard to the outer region will be given below.

[57] Oscillations of comparable amplitude are also present in the North-South colatitudinal field component, as reported by Andrews et al. [2008] and Provan et al. [2009a]. Our findings firstly confirm that these oscillations are generally in phase with the radial field oscillations throughout the inner region, though moving toward lagging quadrature in the outer core near dusk. The implication of the general in-phase behavior is that the field lines are extended outward from the planet where the oscillatory radial field is directed inward, thus weakening the southward directed planetary field, and are contracted inward (relative to the mean) where the radial field is directed outward, thus strengthening the southward field. These relationships are also indicated schematically in Figure 11. Our results also show that the spatial dependency of the amplitude of the colatitudinal field oscillation is rather different to that of the radial and azimuthal components. The field suppression effect in the innermost region is less evident, and there is significantly less variation with LT. Physically, it is evident that the nature of these oscillations is different to that of the equatorial components, the latter being associated with oscillatory tilting of the equatorial quasi-dipolar field lines in the inner region as just discussed, while colatitudinal field oscillations are instead related to changes in the equatorial field strength which must be connected to related changes in the energy density (kinetic or thermal) of the plasma. Indeed, both Provan et al. [2009a] and Khurana et al. [2009] have linked these colatitudinal field oscillations with a rotating LT asymmetry in the ring current plasma. The radial amplitude profile of the colatitudinal field oscillations found here is indeed similar to the radial profile of the quasi-steady equatorial colatitudinal field perturbations produced by the ring current. The colatitudinal ring current field typically peaks at $\sim 10-15 \mathrm{nT}$ near its inner edge at $\sim 7 \mathrm{R}_{\mathrm{S}}$, where the colatitudinal component oscillation amplitude also peaks, and falls near-monotonically to small values at and beyond $\sim 15 \mathrm{R}_{\mathrm{S}}$, as does the colatitudinal oscillation amplitude [Bunce et al., 2007, 2008]. Typically, the oscillations found here correspond to $\sim 20 \%$ of the quasi-steady colatitudinal field perturbations produced by the ring current. Possible physical relations between these oscillations and those of the radial and azimuthal fields will be discussed in section 8 .

[58] In the outer region beyond $\sim 15 \mathrm{R}_{\mathrm{S}}$ the oscillation amplitudes of all field components fall near-monotonically with radial distance, with the largest amplitudes being observed on the nightside in the radial and azimuthal 
components, the former being larger than the latter, with both being larger than that of the colatitudinal component. The phases of the oscillations also show increasing values with radial distance in all components. This trend is already evident within the inner region where the phase gradient is $\sim 1-3^{\circ} \mathrm{R}_{\mathrm{S}}^{-1}$, giving a phase change of $\sim 10^{\circ}-30^{\circ}$ over the radial range between $\sim 5$ and $\sim 15 \mathrm{R}_{\mathrm{S}}$. The trend continues at similar rates in the outer region, where it becomes evident that the gradients are significantly larger on the nightside than the dayside, the associated overall radial phase speeds being $\sim 150 \mathrm{~km} \mathrm{~s}^{-1}$ on the nightside, and $\sim 500 \mathrm{~km} \mathrm{~s}^{-1}$ on the dayside (the latter with large uncertainty estimates). Detailed radial profiles of characteristic wave propagation speeds in the plasma based on field and particle measurements with which these values may be compared have yet to be published. However, estimates based on Cassini density and temperature measurements presented by Wilson et al. [2008] and McAndrews et al. [2009] suggest typical sound and Alfvén speeds within the equatorial ring current region of $\sim 100-400 \mathrm{~km} \mathrm{~s}^{-1}$. Such values are thus at least of the same order as the radial phase speeds deduced here.

[59] The phase lag between the innermost region and $\sim 30 \mathrm{R}_{\mathrm{S}}$ associated with these radial phase speeds is typically $\sim 30^{\circ}$ on the dayside, increasing to $\sim 90^{\circ}$ on the nightside. This LT asymmetry thus implies that the oscillation phase fronts are swept back to earlier LTs with increasing radial distance much more on the nightside than on the dayside, with the consequence that in the outer region they become compressed together in the premidnight sector, and expanded away from each other in the postmidnight sector. This also implies a LT asymmetry in the azimuthal phase speed, with smaller values than the overall angular velocity of the oscillations $\Omega_{M}$ in the premidnight sector, where the azimuthal phase speed is reduced to $\sim 0.7 \Omega_{M}$, and larger angular velocities in the postmidnight sector where the phase speed is increased to $\sim 1.5 \Omega_{M}$.

[60] In addition to the gradual increase in phase with radial distance, the azimuthal field component also shows a much more rapid increase in phase across a narrow layer just outside the core $\left(\sim 15-18 \mathrm{R}_{\mathrm{S}}\right)$ in the LT sector from midnight via dawn to noon, but not in the dusk sector. The azimuthal field oscillations thus remain in approximate lagging quadrature with the radial field oscillations at dusk, while moving toward antiphase in the midnight and noon sectors, and to leading quadrature near dawn. As indicated above, the implication of lagging quadrature with the radial component is that the oscillation field vectors in the equatorial plane rotate with time in the same sense as planetary rotation. Our results show that this sense of polarization is maintained throughout the equatorial region to $30 \mathrm{R}_{\mathrm{S}}$, though moving toward antiphase in the outer region near noon and midnight, except for a restricted LT sector near dawn beyond the core where the equatorial vectors rotate opposite to planetary rotation. We note that antiphase oscillations of the azimuthal and radial field components is characteristic of a NorthSouth oscillating magnetodisc containing a lagging field configuration, while azimuthal oscillations in leading quadrature with the radial, such that the field rotates opposite to planetary rotation, is characteristic of a rotating transverse dipole. The relation between these results will be discussed further below.
[61] Now considering the spatial structure of the oscillatory field in more detail, we note that due to the LT dependencies of the oscillation amplitudes and phases discussed above, particularly the day-night asymmetries, the overall structure of the oscillating fields in the equatorial region depends somewhat on oscillation phase, rather than simply being an almost fixed pattern that rotates around the planet. We first consider $\Phi_{M}=0^{\circ}$, such that the oscillatory field in the equatorial plane points approximately toward the Sun in the inner region. Figure 8 then shows that the equatorial field vectors overall form a partial twin-vortex centered on the nightside, with anticlockwise circulation in the dusk sector, and clockwise (sheared) circulation at dawn. The anticlockwise circulation at dusk relates to the lagging quadrature of the azimuthal component with respect to the radial, but with the radial field being dominant such that the perturbation field lines become convex relative to the planet. The sheared field region at dawn then relates to the rapid increase in phase of the azimuthal component across $\sim 15$ $18 \mathrm{R}_{\mathrm{S}}$, from lagging to leading quadrature with respect to the radial component. The corresponding perturbations in the colatitudinal field component, being approximately in phase with the radial component, are positive (southward) on the dayside and negative (northward) on the nightside within the inner region, while in the outer region negative values are confined to the dusk and midnight sector due to the nightside sweepback of the phase fronts, with positive values being found at all other LTs from dusk.

[62] As the oscillation phase increases to $\Phi_{M}=90^{\circ}$, the pattern rotates such that the vectors in the equatorial plane form an anticlockwise circulation centered near midnight, with dawn-to-dusk magnetic fields in the inner region as expected. However, there is now no corresponding rotated clockwise shear region in the outer dayside sector. The equatorial field vectors in this case may thus be described as forming a single partial anticlockwise vortex centered on the nightside of the planet. With regard to the oscillatory colatitudinal field, this is now generally positive on the dusk side and negative on the dawn side as expected, but negative values extend into the premidnight sector in the outer region (together with positive radial fields) due to the phase front sweep back in this region. With further increase in oscillation phase the pattern then repeats, but with oppositely directed fields at $\Phi_{M}=180^{\circ}$ compared with $\Phi_{M}=0^{\circ}$, and at $\Phi_{M}=270^{\circ}$ compared with $\Phi_{M}=90^{\circ}$. Individual field circulation regions thus appear to form in the dusk sector, anticlockwise when $\Phi_{M} \approx 0^{\circ}$ and clockwise when $\Phi_{M} \approx$ $180^{\circ}$, which rotate to become the dominant circulation at midnight, anticlockwise at $\Phi_{M} \approx 90^{\circ}$ and clockwise at $\Phi_{M} \approx$ $270^{\circ}$, and then expand into the dawn sector to form the anticlockwise and clockwise shear field regions in that sector for $\Phi_{M} \approx 180^{\circ}$ and $\Phi_{M} \approx 270^{\circ}$. The circulation regions finally dissipate in the midmorning sector.

[63] The nature of the oscillatory currents flowing through the equatorial plane in the outer region follows from the description of the equatorial field patterns, with individual current regions emerging near dusk, propagating via midnight, and dissipating postdawn. At $\Phi_{M}=0^{\circ}$ the currents consist of two main regions, northward premidnight and southward postmidnight, each carrying 5 MA, the currents being reversed in sense for $\Phi_{M}=180^{\circ}$. These currents are 
most intense near $\sim 15 \mathrm{R}_{\mathrm{S}}$ where the dominant oscillatory field changes from radial to azimuthal, and are flanked by weaker oppositely directed currents of $\sim 1 \mathrm{MA}$ at dusk and dawn which interface the regions of stronger equatorial field with the weaker fields at large radii and on the dayside. At $\Phi_{M}=90^{\circ}$ three significant current regions are then present, a main northward region of $\sim 5$ MA centered near midnight, and two southward regions of $\sim 2.5 \mathrm{MA}$ at dusk and dawn, which are again reversed in sense for $\Phi_{M}=270^{\circ}$. Overall, the main current regions have the senses expected from the simple hemisphere-to-hemisphere field-aligned current system of Southwood and Kivelson [2007], though the evolution with oscillation phase is somewhat more complicated than the simple rotation envisaged by these authors, with no strong North-South currents flowing from midmorning to postnoon.

[64] If we assume that much of these equatorial NorthSouth currents are indeed associated with field-aligned currents closing in the ionosphere, as seems reasonable, we note that our results imply that the overall current system flowing in the outer region has an associated magnetic dipole moment lying in the equatorial plane that has the same direction as the field within the inner region, associated with the twin-vortex equatorial field system that is generally observed. This is evident for oscillation phases in which the core field points toward or away from the Sun $\left(\Phi_{M} \approx 0^{\circ}\right.$ and $\left.180^{\circ}\right)$, such that two main current regions are present in the outer region, premidnight and postmidnight, with (net) current flowing from north to south in one region and from south to north in the other. However, brief consideration shows that this is also true for phases in which the core field is directed dawn-dusk $\left(\Phi_{M} \approx 90^{\circ}\right.$ and $\left.270^{\circ}\right)$, when three main current regions are present, a region of one sense at midnight, and two near-equal opposite currents at dawn and dusk. A simple estimate of the magnetic moment concerned gives a value of $\sim 10^{25} \mathrm{~A} \mathrm{~m}^{-2}$, about $20 \%$ of the spin-aligned planetary dipole moment. Added vectorially, the combined dipole has a tilt of $\sim 10^{\circ}$ relative to the spin axis, comparable with but smaller than the estimate made by [Southwood and Kivelson, 2007]. The oppositely directed dipole moment of the currents in the core region associated with the field deflection and suppression effect is about a factor of $\sim 50$ smaller than that of the outer currents, due to the smaller current involved and the smaller radial distance of the associated field lines.

[65] An implication of the oscillatory field perturbations in the outer region having the approximate form of a transverse dipole is that the plane of the magnetodisc and plasma sheet in the outer region is tilted in like manner to the magnetic equatorial plane in the inner region described above. That is to say, the magnetodisc current sheet center is displaced southward where the oscillatory radial field is positive, and northward where it is negative, such that overall the northward directed normal of the current sheet is tilted toward the direction of the oscillatory radial field, essentially in the direction of the transverse dipole moment associated with the related current system. Since the radial fields in the outer and inner regions are essentially in phase with each other, allowing for the modest increase in phase in all field components with radial distance discussed above, the implication is that the rotating tilting of the magnetic equatorial plane in the inner region noted above continues into a rotating tilting of the magnetodisc and plasma sheet in the region beyond, as also sketched in Figure 11. The blue dotted line in the figure indicates the undisturbed magnetic equatorial plane which is blown northward of the planetary spin equator beyond $\sim 20 \mathrm{R}_{\mathrm{S}}$ due to the flow of the solar wind relative to the spin axis during the southern summer conditions prevailing at Saturn during the interval studied here [Arridge et al., 2008], while the red dashed line shows the relative position of the magnetic equatorial plane at an instant when the oscillatory equatorial field points from left to right. The magnetodisc is displaced to the north on the left of the diagram, and to the south on the right, as is the magnetic equator in the inner region. In general we also expect the field in the magnetodisc to be swept back out of magnetic meridian planes due to subcorotation of the plasma. That is, the quasi-static fields in this region possess an antiphase relationship between the radial and azimuthal component, with negative azimuthal fields above the center of the disc where the radial field is positive, and positive azimuthal fields below the center of the disc where the radial field is negative. An antiphase oscillation of these fields will thus be introduced in the outer region as the magnetodisc is swept North-South across the spacecraft during the oscillation cycle, which will combine with other oscillations of the same period to move the overall phase toward antiphase, as found in section 3. As noted above, we also illustrate in Figure 11 that at the oscillation phase shown, the oscillatory colatitudinal field is negative (northward) on the left of the diagram and positive (southward) on the right, in phase with the radial component. This then implies a stronger ring current and more stretched out field lines on the left than on the right as illustrated. Our results thus suggest that the current sheet has its most northward deflection about the mean position in the sector with the strongest ring current field, and its most southward deflection in the sector with the weakest ring current field. This is then the opposite of the effect suggested by Khurana et al. [2009], such that this aspect merits further more detailed study.

\section{Discussion of Results}

[66] We first briefly discuss the relation between our results and the modulation of SKR emitted power. As indicated in section 1, SKR is believed to be due to cyclotron maser emission from auroral electrons accelerated into the ionosphere in regions of upward directed field-aligned current [e.g., Zarka, 1998]. The modulation of these currents by the magnetospheric period oscillations investigated here should thus be directly relevant. If the North-South currents we deduce simply flow from hemisphere-to-hemisphere as envisaged by Southwood and Kivelson [2007], then they will be directed upward in one hemisphere, potentially leading to auroral acceleration and SKR emission, and downward in the other, leading to no emission, contrary to the findings of Lamy et al. [2008] that SKR enhancements occur in phase in the two hemispheres. However, as discussed by Andrews et al. [2008] and Provan et al. [2009a], our NorthSouth currents may instead reflect a North-South seasonal asymmetry in a field-aligned current system that has the same sense relative to the ionosphere in the two hemispheres, that would produce no radial and azimuthal fields on the equator if the currents were equal in magnitude. Thus 


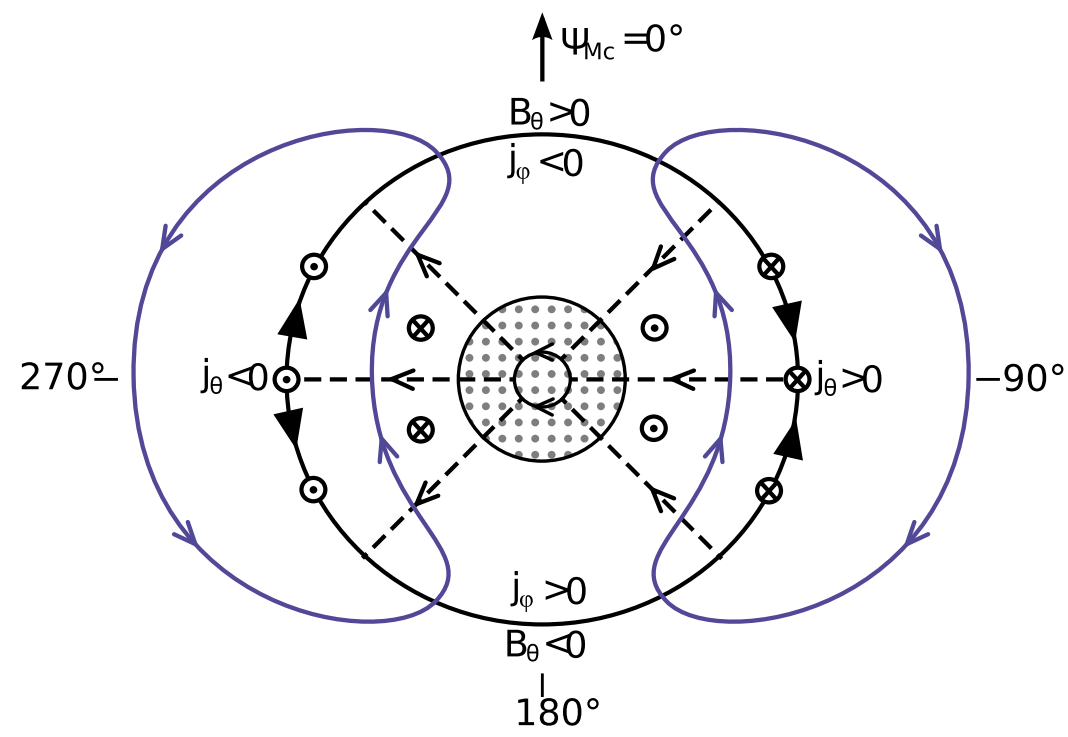

Figure 12. Sketch of rotating currents and fields associated with the oscillatory phenomena observed in Saturn's magnetosphere, viewed looking down on the equatorial plane from the north. The outer solid arrowed black lines indicate the modulation of Saturn's equatorial ring current $\left(j_{\varphi}\right)$, while the radial dashed arrowed black lines indicate the associated field-aligned current flow to and from the ionosphere required by current continuity. The net North-South field-aligned current flow $\left(j_{\theta}\right)$ associated with the hemispheric asymmetry for southern summer conditions is shown by the circled dots for northward current flow (out of the plane of the diagram) on the left of the figure, and by circled crosses (into the plane of the diagram) on the right of the figure. The magnetic perturbations produced in the equatorial field components in the equatorial plane are shown by the solid arrowed blue lines, forming a twin-vortex field system. We also indicate by circled dots and crosses the directions of the inner field-aligned currents associated with the deflection and suppression of the latter fields near the planet. With increasing time this pattern of field and current then rotates anticlockwise around the planet with the magnetospheric period.

counting upward currents relative to the ionosphere as positive for simplicity, if the current is $I_{N}$ in the northern hemisphere and $I_{S}$ in the southern, where $\left|I_{S}\right|>\left|I_{N}\right|$ for the southern summer conditions prevailing during the interval of this study, then we may consider these currents as being due to the sum of a pair of equal currents flowing in the two hemispheres, of magnitude $\left(I_{N}+I_{S}\right) / 2$, and a northward directed hemisphere-to-hemisphere current of magnitude $\left(I_{N}-I_{S}\right) / 2$. The former of these current systems, being antisymmetric about the equator, produces no equatorial field components on the equator, such that the North-South currents deduced here correspond only to the North-South difference current, due to the seasonal interhemispheric asymmetry. In this case, southward directed currents in the equatorial plane imply downward currents in the two hemispheres, with the northern current weaker than the southern, while northward directed currents imply upward currents in the two hemispheres, with the northern current again the weaker for southern summer conditions.

[67] Although this discussion thus makes it clear that we cannot deduce the full field-aligned currents from the NorthSouth equatorial currents estimated here, it suggests a focus on regions of northward directed equatorial current as indicative of upward directed current flows in both hemispheres, certainly in the dominant southern summer hemisphere. Our results then show no obvious simple connection with the SKR modulation, since northward equatorial currents of approximately constant $\sim 6$ MA magnitude are present in the outer region throughout the oscillation cycle, though rotating in local time, e.g., from the postdusk sector when $\Phi_{M}=0^{\circ}$ to the dawn sector when $\Phi_{M}=180^{\circ}$, and so on. As indicated in section 2.2, the maximum SKR power occurs for $\Phi_{M}=210^{\circ}$, when (within the limits of our data coverage) northward currents extend in the outer region from midnight via dawn to dusk (though being weak in the noon sector as usual). However, we note that a specific feature of this and adjacent oscillation phases is a region of strong northward directed current lying at $\sim 15 \mathrm{R}_{\mathrm{S}}$ in the 0600-0800 LT sector, corresponding to the blue starred region in Figures 9e and 9f, that will map to the ionosphere at a latitude of $\sim 73^{\circ}$ [Bunce et al., 2008]. It seems possible that this current region could be the source of the strong high-latitude prenoon SKR emissions reported, e.g., by Galopeau et al. [1995]. With regard to the current densities, as opposed to the area-integrated currents quoted above, we note from Figure 9 that the peak equatorial values are typically $\sim 10 \mathrm{pA} \mathrm{m}^{-2}$ for both northward and southward directed currents, again almost independent of oscillation phase. While our method for determining current densities gives only overall values on scales of $\sim 3 \mathrm{R}_{\mathrm{S}}$ in radius and $\sim 2 \mathrm{~h}$ in LT, and may thus underestimate peak values, we note nevertheless that the above values imply substantial current densities flowing at ionospheric levels, of order $\sim 100 \mathrm{nA} \mathrm{m}{ }^{-2}$. Currents of such magnitude, or higher if 
they represent only the difference current between the two hemispheres as discussed above, are clearly significant in considerations of auroral particle acceleration and wave emission [e.g., Cowley et al., 2004, 2008; Bunce et al., 2008].

[68] Turning now to the nature of the rotating current system implied by our results, we note that it is consistent with the asymmetric ring current picture suggested by Provan et al. [2009a], as indicated schematically in Figure 12. This shows a view looking onto the equatorial plane from the north, with the azimuthally varying ring current $j_{\varphi}$ shown by the solid arrowed black lines. At the oscillation phase depicted, $j_{\varphi}$ is positive at the bottom of the diagram and negative at the top, it being understood that these represent perturbations superposed upon a larger near-constant positive current so that the total current flows consistently eastward as expected. The enhanced positive current at the bottom of the diagram then causes increased outward inflation of the planetary field lines in that LT sector, leading to a decrease in the colatitudinal field, as indicated in the sketch, while the reduced positive current at the top of the diagram similarly leads to an increase in the colatitudinal field relative to the mean. At the instant shown in the sketch, the core phase $\Psi_{M c}$ is thus zero at the top of the diagram (corresponding to the maximum in the $\theta$ field component), and increases clockwise around the planet as indicated, as given by equations (1) and (2a). Current continuity then requires field-aligned currents to flow between the ring current and the ionosphere (central dotted region), that are directed toward the planet on the right of the diagram (about $\Psi_{M c}=90^{\circ}$ ), and away from the planet on the left (about $\Psi_{M c}=$ $90^{\circ}$ ), as indicated by the dashed arrowed black radial lines. If conditions were the same in the northern and southern ionospheres, we would expect equal field-aligned currents to flow in both hemispheres, thus producing no equatorial fields ( $r$ and $\varphi$ field components) lying in the equatorial plane. However, in the northern winter/southern summer conditions that prevailed in the present study, we expect the currents to be greater in the southern hemisphere than in the northern, due to the greater Pedersen conductivity of the former ionosphere compared with the latter. The 'difference' current discussed above is then directed southward (i.e., $j_{\theta}>0$ ) on the right of the diagram and northward (i.e., $j_{\theta}<0$ ) on the left, as also indicated in the diagram. As in the discussion of Southwood and Kivelson [2007], these effective hemisphere-to-hemisphere field-aligned currents then produce the equatorial field shown by the blue arrowed solid lines in the diagram. In the region inside the main northsouth currents, corresponding to the core, the field is directed from the bottom to the top of the diagram, such that the radial component is in phase with the colatitudinal as required, with the azimuthal component being in lagging quadrature with both. We have also included in the sketch a schematic indication of the smaller oppositely directed North-South field-aligned currents flowing in the inner region that lead to the deflection of the core field around the planet as indicated. In the region outside the main North-South currents the equatorial field then forms a twin vortex system as found in our study, akin to the field of a transverse dipole, the dipole concerned being formed by the net hemisphere-to-hemisphere field-aligned current system. A partial ring current system with equal field-aligned currents in the two hemispheres has no associated transverse dipole moment. With regard to the magnitudes of the currents, we note that our results imply effective hemisphere-tohemisphere currents of $\sim 5 \mathrm{MA}$, which in terms of the above discussion implies that the positive and negative modulations in the ring current at the top and bottom of the diagram are at least $\pm 5 \mathrm{MA}$. This compares with estimates by Bunce et al. [2007] of the total dayside ring current, effectively the mean value, of $\sim 10-15 \mathrm{MA}$, with perhaps a further $\sim 5 \mathrm{MA}$ flowing in the plasma sheet to our outer nightside boundary of $30 \mathrm{R}_{\mathrm{S}}$, based on the field strength within the tail lobes. The total averaged field-perpendicular current flowing in the equatorial plane in the region studied is thus $\sim 15-20 \mathrm{MA}$, so that modulations of $\pm 5 \mathrm{MA}$ correspond to remarkably large variations of the equatorial current of $\sim \pm 25-35 \%$.

[69] While this picture thus provides a basic description of the fields observed, we note that it is not obviously consistent with all the details found in our study. In particular, our results show that while the oscillations in the colatitudinal field do not vary greatly in LT, thus indicating the presence of rotating perturbations in the ring current of approximately constant amplitude independent of LT, the oscillations in the radial and azimuthal components do depend strongly on LT, being significantly larger on the nightside than on the dayside. One possible explanation in terms of the above picture, is that while the total fieldaligned current flows to and from the ionosphere required by the varying ring current remain approximately constant independent of oscillation phase, the North-South difference current that leads to the equatorial field components may depend on LT, being smaller on dayside field lines than on nightside field lines. This could happen, for example, if the difference between the Pedersen conductivities of the northern and southern ionosphere is less on the dayside than on nightside.

[70] Finally we briefly comment on the physical origins of these oscillatory phenomena. As discussed in section 1, most theoretical discussion to date has centered on the existence of a corotating convection system that is centrifugally driven by plasma mass-loading in the inner magnetosphere that originates from the emission of water from the moon Enceladus. In terms of the picture presented in Figure 12, we would expect the region with enhanced azimuthal currents to be the outward moving heavy sector, thus corresponding to the region at the bottom of the figure. Dynamically, we would then interpret the equatorial field perturbations shown by the blue lines in the figure as being due to field tilting effects in the presence of differential ionospheric drag between the northern and southern hemispheres, with the drag in the southern summer hemisphere being larger than that in the northern winter hemisphere, as expected. In this case the blue field lines in the sketch then also represent streamlines of the corotating convection system, with the flow being directed opposite to the field. As previously pointed out by Andrews et al. [2008] and Provan et al. [2009a], however, an apparent inconsistency with this picture then concerns the phase of the cold plasma density modulation in the Enceladus plasma torus observed in the inner region $\left(\sim 3-5 \mathrm{R}_{\mathrm{S}}\right)$ by Gurnett et al. [2007], whose heavy sector is in phase with the azimuthal field component, thus peaking at $\Psi_{M c}=90^{\circ}$ on the right of Figure 12, rather 
than being in antiphase with the radial and colatitudinal field components as just outlined. It thus appears that we must suppose the larger-scale flow to be responding primarily to asymmetries that occur at a larger phase angle at larger distances, a suggestion that requires further study to elucidate.

[71] Acknowledgments. Work at Leicester was supported by STFC grant PP/E000983/1. MKD was supported by STFC funding to Imperial College. We thank S. Kellock and the Cassini team at Imperial College for access to processed magnetic field data. We also thank S. Kellett for help in estimating equatorial wave propagation speeds in Saturn's magnetosphere. DJA was supported by a STFC Quota Studentship.

[72] Wolfgang Baumjohann thanks Donald Gurnett and another reviewer for their assistance in evaluating this paper.

\section{References}

Andrews, D. J., E. J. Bunce, S. W. H. Cowley, M. K. Dougherty, G. Provan, and D. J. Southwood (2008), Planetary period oscillations in Saturn's magnetosphere: Phase relation of equatorial magnetic field oscillations and SKR modulation, J. Geophys. Res., 113, A09205, doi:10.1029/ 2007JA012937.

Arridge, C. S., N. Achilleos, M. K. Dougherty, K. K. Khurana, and C. T. Russell (2006), Modeling the size and shape of Saturn's magnetopause with variable dynamic pressure, J. Geophys. Res., 111, A11227, doi:10.1029/2005JA011574.

Arridge, C. S., K. K. Khurana, C. T. Russell, D. J. Southwood, N. Achilleos, M. K. Dougherty, A. J. Coates, and H. K. Leinweber (2008), Warping of Saturn's magnetospheric and magnetotail current sheets, J. Geophys. Res., 113, A08217, doi:10.1029/2007JA012963.

Badman, S. V., S. W. H. Cowley, J.-C. Gérard, and D. Grodent (2006), A statistical analysis of the location and width of Saturn's southern auroras, Ann. Geophys., 24, 3533-3545.

Bunce, E. J., S. W. H. Cowley, I. I. Alexeev, C. S. Arridge, M. K. Dougherty, J. D. Nichols, and C. T. Russell (2007), Cassini observations of the variation of Saturn's ring current parameters with system size, J. Geophys. Res., 112, A10202, doi:10.1029/2007JA012275.

Bunce, E. J., C. S. Arridge, S. W. H. Cowley, and M. K. Dougherty (2008), Magnetic field structure of Saturn's dayside magnetosphere and its mapping to the ionosphere: Results from ring current modeling, J. Geophys. Res., 113, A02207, doi:10.1029/2007JA012538.

Burch, J. L., A. D. DeJong, J. Goldstein, and D. T. Young (2009), Periodicity in Saturn's magnetosphere: Plasma cam, Geophys. Res. Lett., 36 , L14203, doi:10.1029/2009GL039043.

Carbary, J. F., and S. M. Krimigis (1982), Charged particle periodicity in the Saturnian magnetosphere, Geophys. Res. Lett., 9(9), 1073-1076.

Carbary, J. F., D. G. Mitchell, S. M. Krimigis, and N. Krupp (2007a), Electron periodicities in Saturn's outer magnetosphere, J. Geophys. Res., 112, A03206, doi:10.1029/2006JA012077.

Carbary, J. F., D. G. Mitchell, S. M. Krimigis, and N. Krupp (2007b), Evidence for spiral pattern in Saturn's magnetosphere using the new SKR longitudes, Geophys. Res. Lett. 34, L13105, doi:10.1029/2007GL030167.

Carbary, J. F., D. G. Mitchell, S. M. Krimigis, D. C. Hamilton, and N. Krupp $(2007 \mathrm{c})$, Spin-period effects in magnetospheres with no axial tilt, Geophys. Res. Lett., 34, L18107, doi:10.1029/2007GL030483.

Carbary, J. F., D. G. Mitchell, P. Brandt, C. Paranicas, and S. M. Krimigis (2008a), ENA periodicities at Saturn, Geophys. Res. Lett., 35, L07102, doi:10.1029/2008GL033230.

Carbary, J. F., D. G. Mitchell, P. Brandt, E. C. Roelof, and S. M. Krimigis (2008b), Periodic tilting of Saturn's plasma sheet, Geophys. Res. Lett., 35, L24101, doi:10.1029/2008GL036339.

Clarke, K. E., et al. (2006), Cassini observations of planetary-period oscillations of Saturn's magnetopause, Geophys. Res. Lett., 33, L23104, doi:10.1029/2006GL027821.

Connerney, J. E. P., N. F. Ness, and M. H. Acuña (1982), Zonal harmonic model of Saturn's magnetic field from Voyager 1 and 2 observations, Nature, 298, 44-46, doi:10.1038/298044a0.

Cowley, S. W. H., E. J. Bunce, and J. M. O'Rourke (2004), A simple quantitative model of plasma flows and currents in Saturn's polar ionosphere, J. Geophys. Res., 109, A05212, doi:10.1029/2003JA010375.

Cowley, S. W. H., D. M. Wright, E. J. Bunce, A. C. Carter, M. K. Dougherty, G. Giampieri, J. D. Nichols, and T. R. Robinson (2006), Cassini observations of planetary-period magnetic field oscillations in Saturn's magnetosphere: Doppler shifts and phase motion, Geophys. Res. Lett., 33, L07104, doi:10.1029/2005GL025522.
Cowley, S. W. H., et al. (2008), Auroral current systems in Saturn's magnetosphere: comparison of theoretical models with Cassini and HST observations, Ann. Geophys., 26, 2613-2630.

Davis, L., Jr., and E. J. Smith (1990), A model of Saturn's magnetic field based on all available data, J. Geophys. Res., 95(A9), 15,257-15,261.

Desch, M. D., and M. L. Kaiser (1981), Voyager measurement of the rotation period of Saturn's magnetic field, Geophys. Res. Lett., 8, 253-256.

Dougherty, M., et al. (2004), The Cassini magnetic field investigation, Space Sci. Rev., 114(1-4), 331-383.

Dougherty, M. K., et al. (2005), Cassini magnetometer observations during Saturn orbit insertion, Science, 307, 1266-1270, doi:10.1126/ science. 1106098

Espinosa, S. A., and M. K. Dougherty (2000), Periodic pertubations in Saturn's magnetic field, Geophys. Res. Lett., 27(17), 2785-2788.

Espinosa, S. A., D. J. Southwood, and M. K. Dougherty (2003a), Reanalysis of Saturn's magnetospheric field data in view of spin-periodic perturbations, J. Geophys. Res., 108(A2), 1085, doi:10.1029/2001JA005083

Espinosa, S. A., D. J. Southwood, and M. K. Dougherty (2003b), How can Saturn impose its rotation period in a noncorotating magnetosphere?, J. Geophys. Res., 108(A2), 1086, doi:10.1029/2001JA005084.

Galopeau, P. H. M., and A. Lecacheux (2000), Variations of Saturn's radio rotation period measured at kilometer wavelengths, J. Geophys. Res., 105(A6), 13,089-13,101.

Galopeau, P. H. M., P. Zarka, and D. Le Quéau (1995), Source location of Saturn's kilometric radiation: The Kelvin-Helmholtz instability hypothesis, J. Geophys. Res., 100(E12), 26,397-26,410.

Giampieri, G., M. K. Dougherty, E. J. Smith, and C. T. Russell (2006), A regular rotation period for Saturn's magnetic field that may track its internal rotation, Nature, 441, 62-64, doi:10.1038/nature04750.

Goldreich, P., and A. J. Farmer (2007), Spontaneous axisymmetry breaking of the external magnetic field at Saturn, J. Geophys. Res., 112, A05225, doi:10.1029/2006JA012163.

Gurnett, D. A., et al. (2005), Radio and plasma wave observations at Saturn from Cassini's approach and first orbit, Science, 307, 1255-1259, doi:10.1126/science. 1105356

Gurnett, D. A., A. M. Persoon, W. S. Kurth, J. B. Groene, T. F. Averkamp, M. K. Dougherty, and D. J. Southwood (2007), The variable rotation period of the inner region of Saturn's plasma disk, Science, 316(5823), 442-445, doi:10.1126/science.1138562.

Khurana, K. K., D. G. Mitchell, C. S. Arridge, M. K. Dougherty, C. T. Russell, C. Paranicas, N. Krupp, and A. J. Coates (2009), Sources of rotational signals in Saturn's magnetosphere, J. Geophys. Res., 114, A02211, doi:10.1029/2008JA013312.

Krupp, N., et al. (2005), The Saturnian plasma sheet as revealed by energetic particle measurements, Geophys. Res. Lett., 32, L20S03, doi:10.1029/2005GL022829.

Kurth, W. S., A. Lecacheux, T. F. Averkamp, J. B. Groene, and D. A. Gurnett (2007), A Saturnian longitude system based on a variable kilometric radiation period, Geophys. Res. Lett., 34, L02201, doi:10.1029/ 2006GL028336.

Kurth, W. S., T. F. Averkamp, D. A. Gurnett, J. B. Groene, and A. Lecacheux (2008), An update to a Saturnian longitude system based on kilometric radio emissions, J. Geophys. Res., 113, A05222, doi:10.1029/ 2007JA012861.

Lamy, L., P. Zarka, B. Cecconi, R. Prangé, W. S. Kurth, and D. A. Gurnett (2008), Saturn kilometric radiation: Average and statistical properties, J. Geophys. Res., 113, A07201, doi:10.1029/2007JA012900.

Lecacheux, A., and F. Genova (1983), Source localization of Saturn kilometric radio emission, J. Geophys. Res., 88(A11), 8993-8998.

McAndrews, H. J., et al. (2009), Plasma in Saturn's nightside magnetosphere and the implications for global circulation, Planet. Space. Sci., 57, 1714-1722, doi:10.1016/j.pss.2009.03.003

Nichols, J. D., J. T. Clarke, S. W. H. Cowley, J. Duval, A. J. Farmer, J.-C. Gérard, D. Grodentr, and S. Wannawichian (2008), Oscillation of Saturn's southern auroral oval, J. Geophys. Res., 113, A11205, doi:10.1029/2008JA013444.

Provan, G., D. J. Andrews, C. S. Arridge, S. W. H. Cowley, S. E. Milan, M. K. Dougherty, and D. M. Wright (2009a), Polarization and phase of planetary period oscillations on high latitude field lines in Saturn's magnetosphere, J. Geophys. Res., 114, A02225, doi:10.1029/ 2008JA013782.

Provan, G., S. W. H. Cowley, and J. D. Nichols (2009b), Phase relation of oscillations near the planetary period of Saturn's auroral oval and the equatorial magnetospheric magnetic field, J. Geophys. Res., 114 A04205, doi:10.1029/2008JA013988.

Schippers, P., et al. (2008), Multi-instrument analsis of electron populations in Saturn's magnetosphere, J. Geophys. Res., 113, A07208, doi:10.1029/2008JA013098. 
Smith, E. J., L. Davis, D. E. Jones, P. J. Coleman, D. S. Colburn, P. Dyal, and C. P. Sonett (1980), Saturn's magnetic field and magnetosphere, Science, 207, 407-410.

Southwood, D. J., and M. G. Kivelson (2007), Saturn magnetospheric dynamics: Elucidation of a camshaft model, J. Geophys. Res., 112 , A12222, doi:10.1029/2007JA012254.

Warwick, J., et al. (1981), Planetary radio astronomy observations from Voyager-1 near Saturn, Science, 212(4491), 239-243.

Wilson, R. J., R. L. Tokar, M. G. Henderson, T. W. Hill, M. F. Thomsen, and D. H. Pontius Jr. (2008), Cassini plasma spectrometer thermal ion measurements in Saturn's inner magnetosphere, J. Geophys. Res., 113, A12218, doi:10.1029/2008JA013486.
Young, D., et al. (2005), Composition and dynamics of plasma in Saturn's magnetosphere, Science, 307(5713), 1262-1266, doi:10.1126/ science. 1106151 .

Zarka, P. (1998), Auroral radio emissions at the outer planets: Observations and theories, J. Geophys. Res., 103(E9), 20,159-20,194.

D. J. Andrews, S. W. H. Cowley, and G. Provan, Department of Physics \& Astronomy, University of Leicester, University Road, Leicester, LE1 7RH, UK. (dja13@ion.le.ac.uk)

M. K. Dougherty, Blackett Laboratory, Imperial College, London SW7 2BZ, UK. 